Purdue University Purdue e-Pubs

6-1-1995

\title{
Minimizing Communication Overhead for Matrix Inversion Algorithms on Hypercubes
}

Xiaodong Wang

Purdue University School of Electrical and Computer Engineering

Vwani P. Roychowdhury

Purdue University School of Electrical and Computer Engineering

Follow this and additional works at: http://docs.lib.purdue.edu/ecetr

Wang, Xiaodong and Roychowdhury, Vwani P., "Minimizing Communication Overhead for Matrix Inversion Algorithms on Hypercubes" (1995). ECE Technical Reports. Paper 138.

http://docs.lib.purdue.edu/ecetr/138

This document has been made available through Purdue e-Pubs, a service of the Purdue University Libraries. Please contact epubs@purdue.edu for additional information. 


\section{MinIMIZING COMMUNICATION OVERHEAD FOR MATRIX INVERSION ALGORITHMS ON HYPERCUBES}

XIAODONG WANG

VWANI P. ROYCHOWDHURY

TR-ECE 95-17

JUNE 1995

SCHOOL OF Electrical

AND COMPUTER ENGINEERING

PuRdue UnIVERSITY

WEST LAFAYETTE, INDIANA 47907-1285 


\title{
Minimizing Communication Overhead for Matrix Inversion Algorithms on Hypercubes
}

\author{
Xiaodong Wang ${ }^{1}$ and Vwani P. Roychowdhury ${ }^{2}$ \\ School of Electrical and Computer Engineering \\ Purdue University \\ West Lafayette, IN 47907, USA
}

1 xiaodong@ecn.purdue.edu
2 vwani@ecn.purdue.edu 


\begin{abstract}
The mirin contribution of this report is the development of novel algorithms that make efficient use of the communication system in distributed memory architectures with processing elements interconnected by a hypercube network. These algorithms achieve almost optimal overlap of communication delays by computation, leading to a minimization of communication overhead. Rigorous analytical and numerical performance analysis of our parallel algorithms are presented as well. The parallel algorithm under study in this report is the parallel Gauss-Jordan matrix inversion algorithm. Many of the ideas introduced in this report, however, apply to other linear algebra algorithms as well.

Parallel Gauss-Jordan matrix inversion algorithms on the hypercube multiprocessors have been extensively studied in the literature. Two common data partitioning strategies for matrix algorithms are row-wise partitioning and submatrix partitioning. It has been claimed that for the parallel G.J inversion algorithm, submatrix partitioning scheme exhibit communication overhead advantages not shared by partitions limited to rows or columns. Most parallel algorithms proposed in the literature, however, do not attempt to overlap inter-processor communication by computation. As a result, the formula execution time=computation time + communication time is used to analyze the complexity of the parallel algorithm. However, during most of the communication time, the processors are actually idle, waiting for the data to arrive.

Most commercially available parallel machines provide communication interrupt handling capability. By utilizing this feature, we believe a lot of parallel matrix algorithms can be improved by overlapping interprocessor communication and computation. In this report we propose and analyze new parallel GJ inversion algorithms under different data partitioning strategies, with or without partial pivoting.

We first propose a new broadcasting algorithm on the hypercube multiprocessor for parallel GJ algorithm. This algorithm ensures that the data are sent out from the source and arrives at the destinations at the earliest possible time. We then give the parallel GJ inversion algorithm using row partitioning. The strategy to overlap communication by computation is for each processor to compute and send out the data needed by the other processors as early as possible. We prove a lower bound on the matrix size such that data transmission is fully overlapped by computation. We also prove that the message length in the input buffer of each processor is art most 2.

We also consider the algorithms under submatrix partitioning, with or without pivoting. We show that when submatrix partitioning is used, even when the communication is fully overlapped by computation, the communication overhead is larger than when using row partitioning. Thus, we show that by minimizing communication overhead, the row partitioning scheme can indeed have better overall performance than the submatrix partitioning scheme.

Finally we extend the idea of overlapping communication and computation to the parallel LU factorization algorithm.
\end{abstract}




\section{Contents}

1 Introduction 1

1.1 Assumptions on the Machine Model . . . . . . . . . . . . . . . . . 1

1.2 Algorithmic Communication Model . . . . . . . . . . . . . . . . . 2

1.3 Related Work on Parallel Gauss-Jordan Matrix Inversion Algorithm . . . . . . . 3

1.4 Motivation for a New GJ Inversion Algorithm . . . . . . . . . . . . . . . . . 4

1.4.1 Sequential GJ inversion algorithm with column interchanges . . . . . . . . 4

1.4.2 A brief review of the subcube-grid parallel GJ inversion algorithm in [1] . . 4

1.5 Sitrategy for Overlapping of Communication and Computation . . . . . . . . 5

$\begin{array}{lll}2 & \text { Summary of Results } & 7\end{array}$

3 Algorithm I: Row Partitioning With Pivoting 9

4 The SBT; Broadcasting Algorithm $\quad 11$

5 Analysis of Algorithm I 17

6 Algorithm II: Submatrix Partitioning, Without Pivoting 29

7 Analysis of Algorithm II $\quad 33$

$8 \quad$ Algorithm III: Submatrix Partitioning, With Pivoting 37

9 Scalability Analysis $\quad 39$

9.1 The Isoefficiency Metric of Scalability $\ldots \ldots \ldots$. . . . . . . . . . . . . . 39

9.2 The Isospeed Metric of Scalability . . . . . . . . . . . . . . . . . 39

10 LU Factorization $\quad 41$

11 Concluding Remarks $\quad 45$ 


\section{Introduction}

One of the challenges for large-scale parallel processing is to minimize commnnication overhead. Although one can attempt to reduce it via architectural innovations, physical reality dictates that remote access will always be significantly slower than local access. Software techniques for reducing overhead are therefore essential.

An efficient approach to reducing communication overhead is to allow communication to overlap computation. This helps reducing overhead in two ways: First, when one process is waiting for data from a remote process, another ready process maybe scheduled for execution. Secondly, even a single process may wait for multiple data items simultaneously, and continue execution whenever any of the expected items arrive [32]. Research projects addressing overlap of communication and computation have been carried out at both hardware and software levels. Research prototypes of message driven architectures, such as the J-Machine [33] and Monsoon [34], expend a sigificant amount of hardware to integrate communication with compntation. The message driven execution style promotes the overlap of communication and computation, and exhibits impressive communication performance. For the current message passing architectures, a communication mechanism, active message, has been proposed [31]. This model minimizes the software overhead in message passing mirchines and achieves overlap of communication and computation.

The approaches mentioned above deal with the communication overhead from a general engineering perspective. We, however, in this report, treat the problem of minimizing communication overhead from an algorithmic perspective. That is, given a machine model and a computational task, how can one develop an efficient parallel algorithm which achieves overlap of communication and computation, and incurs minimum communication overhead. The technique used in this report is analytic, which provides rigorous quantitative evaluation on the performance of the parallel algorithm. We consider the parallel Gauss-Jordan matrix inversion algorithm on message passing hypercube multiprocessors.

\subsection{Assumptions on the Machine Model}

Throughout this report, we make the following assumptions about the machine model:

1. The parallel machine has binary-cube network with all-port communication capability, i.e., the lardware supports simultaneous communication on all the channel:;. Machines with hypercube network that support all-port communication include CM-2, Chi-200 and nCUBE 2 .

2. It is a multiple instruction multiple data (MIMD) parallel machine. MIMI) machines include nCUBE 2, iPSC, CM 2, Paragon, etc.

3. The interprocessor communication is through DMA controlled asynchronous message passing. Machines with this feature include nCUBE 2, iPSC, Paragon, etc. 
4. The software supports user specified interrupt handling. Intel iPSC, Intel Paragon and CM 5 have this feature.

\subsection{Algorithmic Communication Model}

The most common cost model used in algorithm design for large-scale multiprocessor assumes that the program alternates between computation and communication phases, and that communication requires time linear in the size of the message, plus a setup cost. Consider a simple scenario in which all the processors have the same computation and communication loads, and the program alternates between computation and communication phases in a synchronous manner. Thus, the time to run a program is $T=T_{\text {compute }}+T_{\text {communicate }}$ and $T_{\text {communicate }}=\sum_{i=1}^{N_{c}}\left(t_{s}+t_{w} L_{i}\right)$, where $t_{s}$ is the setup cost, $t_{\boldsymbol{w}}$ is the time per byte, $\mathrm{L}$; is the $\boldsymbol{i}^{\text {th }}$ message length, and $N_{c}$ is the total number of communications. To achieve $90 \%$ of the peak processor performance, the algorithm must be tailored to achieve a sufficiently high ratio of computation to communication, i.e., $T_{\text {compute }} \geq 9 T_{\text {communicate }}$. A high performance network is required to minimize the communication time, and it sits $90 \%$ idle [31].

If communication and computation are fully overlapped, the situation is very different. The time to run a program becomes $\mathrm{T}=\max \left(T_{\text {compute }}+N_{\mathrm{c}} t_{s}, \sum_{i=1}^{N_{c}} t_{w} L_{i}\right)$. Thus, to achieve high processor efficiency, the data transmission and computation time need only balance, and the computation time need only swamp the setup overhead, i.e., $T_{\text {compute }} \gg N_{c} t_{s}[31]$.

Next we introduce the communication model used this report, and the concept of full overlap of communication and computation. Assuming that at time $T_{1}$, processor $P_{1}$ starts sending a message of length $m$ to its adjacent processor $P_{2}$ (i.e., $P_{1}$ and $P_{2}$ is directly connected by communication channel). $P_{\mathbf{1}}$ will first spend time t, to set up the communication channel. The message will then take additional time $t_{w} m$ to reach $P_{2}$, i.e., the message arrives at $P_{2}$ at time $T_{1}+\mathrm{t}, t_{w} m . P_{1}$ can return to computation after setting up the communication channel, i.e., at time $T_{\mathbf{1}}+\mathrm{t}$,. Now assume that at time $T_{2}$, processor $P_{2}$ needs to read this message from $P_{1}$. If this message has already been in the input buffer of $P_{2}$, i.e., this message was sent out by $P_{1}$ at time $T_{1}$ such that $T_{1}+\left(\mathrm{t},+t_{w} m\right) \leq T_{2}$, then $P_{2}$ can read this message without any delay. Here we assume it takes negligible time to read the message in the input buffer. Otherwise, $P_{2}$ will rernain idle until the message arrives in its input buffer. The idle time is $t_{i d l e}=T_{1}+\left(t_{s}+t_{w} m\right)-T_{2}$. Therefore the communication overhead of each processor contains two parts: the setup time, when the processor sets up the communication channel, and the idle time, when the processor is idle, waiting for message to arrive.

In a parallel computation process on a multiprocessor, if for every processor, the idle time is always zerı throughout the process (that is, each processor is either doing computation, or setting up the communication channel, but never idle), then we say communication is fully overlapped by computation in this process. Hence our objective is to accomplish this full overlap of communication and computation, and at the same time, to minimize the total setup overhead. 


\subsection{Rellated Work on Parallel Gauss-Jordan Matrix Inversion Algorithm}

Matrix operation, such as inversion and factorization, is a standard part of any linear algebra library. Such libraries are critical for the evolution of parallel computer into useful tools for large scale scientific computations. Two common sequential algorithms for dense matrix inversion are Gaussian :Elimination (GE) and Gauss-Jordan (GJ) Elimination. They have the same operation count (FLOPs) for inversion. It has been shown that GJ is significantly more efficient in parallel than GE, because parallel GJ has a more homogeneous work load distribution and incurs less communication overhead [12]. Furthermore, GJ inversion is an in-place matrix inversion algorithm. Thus no additional memory is required as work place during the process of GJ elimination.

Several parallel algorithms have been developed for implementing GJ elimination with or without pivoting on distributed-memory architectures. Some of them are fine-grain algorithms developed for hypercubes assumed to have little setup cost and high communicatioa bandwidth, e.g., the Caltech Hypercube $[11,12]$. The others are medium-grain algorithms developed for hypercubes with substantial message passing latency, e.g., the commercially available Intel iPSC/1, iPSC/2, iPSC/860 and nCUBE 2 hypercubes [1, 17]. In [1] some of the algorithms representing recent developments in both classes are reviewed.

It was shown in [2] that the GJ algorithm has essentially different properties when using column interchanges instead of row interchanges for improving numerical stability. A more satisfactory bound for the residual norm can be derived for solutions obtained by GJ with column interchanges instead of row interchanges. In [1] a medium-grain parallel algorithm for implementing GJ inversion with colurnn interchanges on a hypercube multiprocessor was proposed. The hypercube network was configured as a two-dimensional subcube-grid to support submatrix partitionings. The authors claimed that for GJ inversion with column interchanges on hypercubes with substantial communication latency, submatrix partitions had communication advantages not shared by partitions limited to rows or columns. In their algorithm, no attempt was made to overlap communication and computation.

Two popular optimization techniques applied to parallel matrix computations for improving concurrence are the send-ahead strategy that attempt to communicate data as early as possible, and the compute-and-send-ahead strategy that computes in advance data which have to be communicated $[3,4,5]$. Their most important aim is to avoid the processors idleness due to the data waiting tirne by overlapping communication and computation. In [3] these two optimization strategies were applied to parallel LU decomposition algorithms, with or without partial pivoting. In their implementation, they used the communication subroutine provided by the software, and it is not clear how the overlap is achieved. No analytical performance analysis was provided in their paper. Their experimental results also showed the superiority of the square scattered decomposition strategy. 


\subsection{Motivation for a New GJ Inversion Algorithm}

\subsubsection{Sequential GJ inversion algorithm with column interchanges}

We first give the sequential G J algorithm with column interchanges for inverting an $\mathrm{N}$ x $\mathrm{N}$ matrix $\mathrm{A}=\left[\boldsymbol{a}_{i, j}\right]$. This algorithm performs in-place inversion. An array $\boldsymbol{\sigma}_{1: N}$ is used to record the permutation of the columns. Initially $\boldsymbol{a} ;=\dot{\mathrm{z}} \boldsymbol{i}=1,2, \cdots, \mathrm{N}$. At the k-th step of GJ reduction, if the pivot column is found to be column pivot, then columns $\mathrm{k}$ and pivot need to be interchanged, and we record this interchange by swapping the value of $\boldsymbol{\sigma}_{\boldsymbol{k}}$ and $\boldsymbol{\sigma}_{\text {pivot }}$. Note that in the algorithm we actually do not interchange columns, but rather, we just address the permuted columns using the information saved in $\boldsymbol{a}$. In this way, some intermediate data movement can be saved. As a result, $\boldsymbol{A}$ is overwritten by a row and column permuted form of $A^{-1}$. Let $\mathrm{B}=\left[\boldsymbol{b}_{\boldsymbol{i}, j}\right]=A^{-\mathbf{1}}$, then after applying the algorithm to A, $\boldsymbol{a}_{i, j}=\boldsymbol{b}_{k, l}$, where $\mathrm{k}=\boldsymbol{a}$; , and $\boldsymbol{\sigma}_{l}=j$.

$$
\begin{aligned}
& \text { for } \mathrm{k}=1 \text { to } \mathrm{N} \\
& \text { pivot }=k \\
& \text { for } j=k+1 \text { to } N \\
& \text { if }\left|\boldsymbol{a}_{k, \sigma_{\mathrm{j}}}\right|>\left|\boldsymbol{a}_{k, \sigma_{\text {pivot }}}\right| \text { then pivot }=\mathbf{j} \\
& \sigma_{k} \longleftrightarrow \sigma_{\text {pivot }} \\
& \text { for } \mathbf{j}=1 \text { to } \mathrm{N} \text { and } \mathbf{j} \neq \mathrm{k} \\
& a_{k, \sigma_{j}}=a_{k, \sigma_{j}} / a_{k, \sigma_{k}} \\
& \text { for } \mathrm{i}=1 \text { to } \mathrm{N} \text { and } \mathrm{i} \neq \mathrm{k} \\
& a_{i, \sigma_{j}}=a_{i, \sigma_{j}}-a_{i, \sigma_{k}} * a_{k, \sigma_{j}}
\end{aligned}
$$

for $i=1$ to $\mathrm{N}$ and $\mathrm{i} \neq k$

$$
\begin{array}{r}
a_{i, \sigma_{k}}=-a_{i, \sigma_{k}} / a_{k, \sigma_{k}} \\
a_{k, \sigma_{k}}=1 / a_{k, \sigma_{k}}
\end{array}
$$

\subsubsection{A brief review of the subcube-grid parallel GJ inversion algorithm in [1]}

We next briefly review the GJ inversion algorithm proposed in [1] (Thereby we call this algorithm Algorithm 0 ). The matrix is partitioned into submatrices, and the data are distributed among the processors using full column and row wrap-mapping. Each column and each row of processors form a subcube. Within the main loop, there are three blocks of code corresponding to the communication of the pivot row; the selection and permutation of the pivot column; and the GJ elimination on the processor's share of matrix.

A hypercube of dimension $d$ is configured as a $\gamma_{1} \times \gamma_{2}=p=2^{d}$ subcube-grid. Each subcubecolumn has dimension $\boldsymbol{d}_{\mathbf{1}}=\log \gamma_{\mathbf{1}}$ and each subcube-row has dimension $\boldsymbol{d}_{2}=\log \gamma_{\mathbf{2}} . \boldsymbol{d}_{\mathbf{1}}$ and $\boldsymbol{d}_{\mathbf{2}}$ differ at most by one for optimal performance. At each iteration, there are two types of communication 
required for this algorithm: one to broadcast the pivot row and another to find and broadcast the pivot column. The former involves one-to-all broadcast within the subcube-column, and the latter involves permutations within the subcube-row. After every processor receives the appropriate pivot row segment, they each determine the local pivot element and the corresponding pivot column. Every processor has the pivot element and the corresponding segment of the final pivot column after permutations (recursive doubling) within the subcube-row. Since recursivr: doubling within a subcube of dimension $\boldsymbol{d}_{2}$ involves $\boldsymbol{d}_{2}$ steps of write-read pairs, this portion of the algorithm causes a synchronization barrier for all processors. No processor can start computation before the entire communication is completed. Therefore this algorithm is implemented in a synchronous manner, i.e., in every iteration, a processor starts performing its computation only after the entire data communication is completed.

Assuming that $\gamma_{\mathbf{1}}=\gamma_{\mathbf{2}}=\sqrt{p}$, then the communication of the pivot row amounts $\boldsymbol{d}_{\mathbf{1}}=\frac{\mathbf{1}}{\mathbf{2}} \log \mathrm{p}$ one-hop transfers of a message of size $N / \sqrt{p}$. The selection and permutation of the pivot column amounts to $\boldsymbol{d}_{\mathbf{2}}=\frac{\mathbf{1}}{\mathbf{2}} \log p$ one-hop exchanges of a message of size $N / \sqrt{p}$. Therefore, the total communication overhead for each processor incurred in this algorithm is $T_{\text {comm }}=N\left(d_{1}+d_{2}\right)\left(t_{s}+\right.$ $\left.t_{w} \frac{N}{\sqrt{p}}\right)=N \log p\left(t_{s}+t_{w} \frac{N}{\sqrt{p}}\right)$.

Since no communication is overlapped by computation, $T_{\text {comm }}$ appears as a whole in the total execution time of the algorithm, i.e., $\mathrm{T}=T_{\text {comp }}+T_{\text {comm }}$, where $T_{\text {comp }}$ is the total computation time of each processor.

\subsection{Strategy for Overlapping of Communication and Computation}

The strategy for overlap of communication and computation is to let each processor to compute and send out the data needed by the other processors as early as possible, so that these data would have arrived at the destinations when they are to be used. This is called compute-and-send-ahead strategy and we will elaborate on it later when we give the parallel GJ inversion algorithms. The best we can do to this end is to make the idle time of each processor zero, and to make the total setup overhead of each processor as small as possible.

In [3] the send-ahead and the compute-and-send-ahead strategies have been applied to the parallel LU factorization algorithm to achieve overlap of communication and computation. The main communication operation of the algorithm is broadcasting the pivot rows and the multipliers. Since the data is expected to arrive at the destinations at the earliest possible time, the data broadcast process is of the most importance and should be carefully designed. However, in their paper, the authors tcook the broadcast within a subcube (which sends data from one processor to all others within the subcube) as a communication primitive, and did not specify the details of this process. And no analytical performance analysis was given for the algorithms in the paper.

To accomplish full overlap of data transmission by computation, we need the high level languages for the parallel machines to provide the interrupt handling capability. Whenever a message arrives at the input buffer of a processor, the processor is interrupted from the current computation and 
starts executing the interrupt handling subroutine, which involves forwarding the message to some adjacent processors. After that the processor resumes the previous interrupted computation. This feature guarantees that the message is passed along the communication tree in the hypercube without delay and reaches the destinations at the earliest possible time. For example, for one-to-all broadcasting, when the message arrives at a processor, and if this processor is not a leaf node of the broadcasting tree, then this processor is interrupted from the current computation and forwards the message to the adjacent nodes on the hypercube which has not got the message, before it resumes the interrupted computation. This feature of interrupt handling is crucial for the overlap of communication and computation. Throughout this report we assume the underlying machine model has this feature. 


\section{Surnmary of Results}

In this report, we show that by utilizing the interrupt handling capability of the parallel machine, a set of asynchronous algorithms can be designed such that the data transmission can be partially or fully overlapped by computation. We propose a new one-to-all broadcast algorithm on hypercube that helps to achieve overlap of communication and computation for the parallel GJ inversion algorithm.

We give the new parallel GJ inversion algorithms under different data partitioning strategies (i.e., row partitioning and submatrix partitioning), with or without partial pivoting. For each algorithm, we prove a lower bound on the matrix size such that data transmission is fully overlapped by computation, and the total communication overhead when full overlap is achieved. Our conclusion is that when the matrix size is large enough such that data transmission is fully overlapped by computation, ihe row partitioning strategy has the lowest communication overhead. We also give the scalability analysis for the algorithms discussed in this report. Furthermore, we also apply the idea of overlap communication and computation to the parallel LU factorization algorithm.

\begin{tabular}{|c||c|}
\hline Partition Strategy & Total Comm. Overhead \\
\hline \hline row partitioning & $N\left(t_{s}+t_{w} N\right) \log p$ \\
\hline submatrix partitioning & $N\left(t_{s}+t_{w} \frac{N}{\sqrt{p}}\right) \log p$ \\
\hline
\end{tabular}

Table 1: The communication overhead for the parallel GJ inversion algorithm under the row partitioning and submatrix partitioning, when no overlap of communication and computation is attemped. The matrix size is $\mathrm{N} \mathrm{x} \mathrm{N}, t_{s}$ is the communication startup overhead, $t_{w}$ is the data transmission rate, $\mathrm{p}$ is the number of prossors.

\begin{tabular}{|c||c|c|}
\hline Partition Strategy & Condition & Total Comm. Overhead \\
\hline \hline row & $\left(\frac{N}{p}-3\right) N f \geq \frac{1}{2} p t_{s}+2\left(t_{s}+t_{w} N\right) \log p$ & $\frac{1}{2} N t_{s}$ \\
\hline submatrix, w/o pivoting & $\left(\frac{N^{2}}{p}-\frac{3 N}{\sqrt{p}}\right) f \geq 2 \sqrt{p} t_{s}+2\left(t_{s}+t_{w} \frac{N}{\sqrt{p}}\right) \log p$ & $N t_{s}$ \\
\hline submatrix, w/ pivoting & - & $\frac{1}{2} N(1+\log p) t_{s}$ \\
\hline
\end{tabular}

Table 2: Summary of key theoretical results in this report, for the conditions of full overlap of communication by computation, and the total communication overhead of the algorithm when full overlap is achieved, under the row or submatrix partitioning, with or without pivoting.

Table I. lists the communication complexities for the parallel GJ inversion algorithm under the row partitioning and submatrix partitioning, where no overlap of communication and computation is attempted $[1,9]$. Table 2 summarizes some theoretical results in this report on the conditions of full overlap and the total communication overhead when frill overlay is achieved.

Figure 1 depicts some numerical results on the total communication overhead of different algo- 
rithms. Note that the vertical axis is log scaled. It is clear from this plot that overlapping data communication and computation can greatly reduce the communication overhead of the parallel algorithm. Furthermore, when the matrix size is large enough, row partitioning is superior to submatrix partioning.

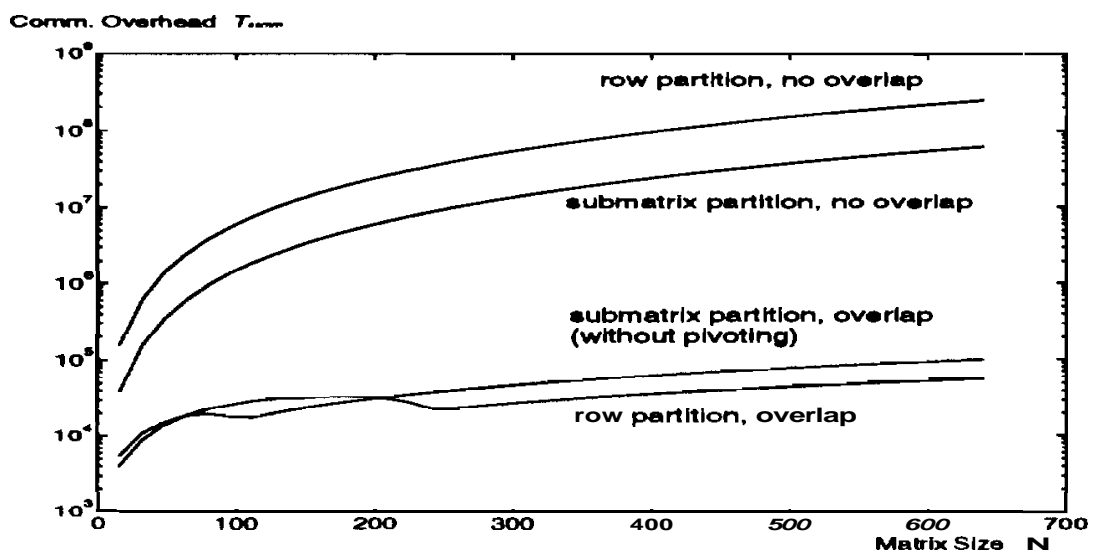

Figure 1: Comparison of the communication overheads of four parallel GJ matrix inversion algorithms under the row or submatrix partitioning, with or without overlap (Number of processors $p=16$ ).

This report is organized as follows: In section 3 we give our parallel GJ inversion algorithm using row partitioning, with pivoting (Algorithm I). In section 4 we propose a new one-to-all broadcast algorithm on hypercube that helps to achieve overlap of communication and computation. In section 5, we give the performance analysis of Algorithm I. In section 6 we give Algorithm II, which uses submatrix partitioning and without pivoting. The analysis of Algorithm II is given in section 7. In section 8, we discuss Algorithm III, which also uses submatrix partitioning but with pivoting. In section 9, we give the scalability analysis of our parallel algorithms. In section 10 we extend the idea of overlapping communication and computation to parallel LU factorization algorithm. Section 11 concludes this report. 


\section{Algorithm I: Row Partitioning With Pivoting}

In this section, we give our algorithm of parallel GJ inversion with column interchanges, using row partitioning. Suppose we want to invert an $\mathrm{N} \times \mathrm{N}$ matrix $\mathrm{A}$ on a dimensional hypercube containing, $\mathrm{p}=\mathbf{2}^{\boldsymbol{d}}$ processors. Assume $\mathrm{N}=\mathrm{np}$. We partition the matrix by rows and use wrapmapping to distribute the rows of A among processors, i.e., rows $\mathrm{k}, \mathrm{p}+\mathrm{k}, 2 p+\mathrm{k}, \cdot \ldots, \mathrm{N}-\mathrm{p}+k$ are assigned to processor $P_{k}, 1 \leq \mathrm{k} \leq \mathrm{p}$. We will discuss how to determine the physical address of processor $P_{k}$ on the hypercube in the next section. We use notation $[\mathrm{k}]$ to represent $[(\mathrm{k}-1) \bmod$ $p]+1$, thus $1 \leq[\mathrm{k}] \leq p$.

When the matrix is partitioned by rows, each row is entirely within one processor. Thus the search for the pivot element can be done by the single processor which has the pivot row, and the recursive tloubling is no longer needed. In the algorithm, during the k-th loop, each processor first gets the b-th pivot row of A and the pivot element from pro essor $P_{[k]}$, and then updates all the rows assigned to it. In order to overlap the communication by computation, the pivot row should be sent out at the earliest possible time, so that when a processor is to use it, it would have already arrived at that processor. Since processor $P_{[k+1]}$ is assigned row $(k+1)$ of $A$, we let it update this row a.t first during the k-th loop. Then it finds the pivot element and normalizes this row (cornpule-ahead). After that it broadcasts this $(\mathrm{k}+1)$ st pivot row and the pivot element to all the other processors, before it resumes to update the other $n-1$ rows using the $\mathrm{k}$-th pivot row (send-ahead). By using this cornpule-and-send-ahead strategy, the $(k+1)$ st pivot row is transmitted through the communication channels while all the processors are still updating in the kth loop. Thus the overlap of communication and computation is achieved.

Since the message may take several hops to reach the destinations, it needs to be routed to the next processor without delay upon arrival at an intermediate processor. This is realized by a local message routing algorithm and the communication interrupt handling capability of the machine. By the local rnessage routing algorithm, each processor knows where to forward the: incoming message to. We will discuss this algorithm in the next section. Whenever a message arrives at a processor, the processor is interrupted from the computation. By identifying the header of the message the processor knows which pivot row this message contains. It will then determine where to forward this message to. If it does not need to forward it, it resumes its computation. Otherwise, it has to forward the message to some of its adjacent processors, and incurs a setup overhead oft,, before it resumes its computation. If after completing the $k$-th loop of updating, the $(k+1)$ st pivot row still has not arrived at the processor, then the processor has to wait for it before it can start the $(\mathrm{k}+1)$ st updating loop.

The following is pseudo code for the kth step of the parallel GJ inversion algarithm with column interchanges, using row partitioning:

\footnotetext{
if $\left(\mathrm{P}=: P_{[k+1]}\right)$ then

read in the kth pivot row and pivot element;
} 
upclate the $(k+1)$ st row of $A$;

find the $(\mathrm{k}+1)$ st pivot element;

normalize the $(\mathrm{k}+1)$ st pivot row;

broadcast the $(\mathrm{k}+1)$ st pivot row;

upclate the rest $(n-1)$ rows of $A$;

if $\left(\mathrm{P}=: P_{[k]}\right)$ then

updlate the $(\mathrm{n}-1)$ rows of A (other than the kth row);

if $\left(P \neq P_{[k+1]}\right.$ and $\left.P \# P_{[k]}\right)$ then

read in the kth pivot row and pivot element;

update the $\mathrm{n}$ rows of $\mathrm{A}$;

\section{Intemrpt handling:}

read the incoming data message;

forward the incoming message to appropriate processors, if needed; 


\section{The SBT; Broadcasting Algorithm}

In order to implement the compute-and-send-ahead strategy discussed in the previous section, we need to construct a set of $\mathrm{p}$ broadcasting trees in the hypercube. At the kth broadcasting in Algorithm I, the root of the tree is $P_{[k]}$. Furthermore, each broadcasting tree should have the following two features:

1. $P_{[k+1]}$ and $P_{[k]}$ should be directly linked.

As discussed in the previous section, the $(k+1)$ st row of matrix A is broadcast by processor $P_{[k+1]}$ immediatedly after it is updated and normalized in the $k$ th stage of the parallel GJ inversion algorithm, before $P_{[k+1]}$ starts updating the other rows using the: $k$ th pivot row. To broadcast the $(\mathrm{k}+1 t)$ st at the earliest possible time, processor $P_{[k+1]}$ should receive the $k$ th pivot row from processor $P_{[k]}$ at the earliest possible time. This implies that $P_{[k+1]}$ and $P_{[k]}$ should be adjacent on the hypercube.

2. $P_{[k+1]}$ should be a leaf node in the kth broadcasting tree.

After receiving the kth pivot row, $P_{[k+1]}$ should not be involved in forwardling the message to some other processors, so that its computation is not delayed by the startup time overhead, and the $(k+1)$ st row can be sent out at the earliest possible time. This implies that $P_{[k+1]}$ should be a leaf node in the $k$ th broadcasting tree.

We next discuss how to determine the physical address of each processor on the hypercube. Since the logically consecutively numbered processors need to be physically adjacent, i.e., processors $P_{[k+1]}$ and $P_{[k]}$ be adjacent on the hypercube, we address the processors in a binary-reflected Gray code order with the starting address as $\mathbf{0}$ [7]. Let the d-bit code of $\mathrm{p}=\mathbf{2}^{\boldsymbol{d}}$ integers be $\operatorname{Gray}(\boldsymbol{d})$. The binary-reflected Gray code on d bits is recursively defined as follows.

Let $\operatorname{Gray}(d)=\left(g_{1}^{d}, g_{2}^{d}, \cdots, g_{2^{d}-1}^{d}, g_{2^{d}}^{d}\right)$. Then

$$
\operatorname{Gray}(d+1)=\left(0 g_{1}^{d}, 0 g_{2}^{d}, \cdots, 0 g_{2^{d}}^{d}, 1 g_{2^{d}}^{d}, 1 g_{2^{d}-1}^{d}, \cdots, 1 g_{1}^{d}\right)
$$

Alternatively,

$$
\operatorname{Gray}(d+1)=\left(g_{1}^{d} 0, g_{1}^{d} 1, g_{2}^{d} 1, g_{2}^{d} 0, g_{3}^{d} 0, g_{3}^{d} 1, \cdot \cdot, g_{2^{d}}^{d} 1, g_{2^{d}}^{d} 0\right) \text {. }
$$

We use this binary-reflected Gray code mapping to associate each processor's logical address with its physical address, i.e., let the physical address of processor $P_{i}$ be $\boldsymbol{g}_{i}^{d}$. For the rest of the report, we will just use $P_{\boldsymbol{i}}$ to denote the physical address $\boldsymbol{g}_{\boldsymbol{i}}^{\boldsymbol{d}}$.

In [7] the spanning binomial tree (SBT) of a d-cube rooted at node $s$ is defined as follows. Let $\boldsymbol{i}$ be any node, and let $\boldsymbol{c}=\boldsymbol{i} \oplus \boldsymbol{s}$, where $\oplus$ denotes bitwise exclusive OR operation. Let q be such that $\boldsymbol{c}_{q}=1$ and $\boldsymbol{c}_{\boldsymbol{m}}=\mathbf{0}, \boldsymbol{V} \boldsymbol{m} \in \boldsymbol{M}(\boldsymbol{c})=\{\mathrm{q}+1, q+2, \ldots, \mathrm{d}-1\}$, and let $\mathrm{q}=-\mathrm{l}$ if $\boldsymbol{c}=\mathbf{0}$. The set $M(c)$ is the set of leading zeros of $\boldsymbol{c}$. Then in $\operatorname{SBT}(\boldsymbol{s})$, the children nodes and the parent node of a given node $i$ are

$$
\text { children }(i)=\left\{\left(i_{d-1} i_{d-2} \cdot . \bar{i}_{m} \ldots i_{0}\right)\right\}, V m \in M(c)
$$




$$
\operatorname{parent}(i)= \begin{cases}\phi, & i=s \\ \left(i_{d-1} i_{d-2} \cdots \bar{i}_{q} \ldots i_{0}\right), & i \neq s\end{cases}
$$

It can be easily verified that the children and parent functions defined above are consistent, i.e., that node $j$ is a child of node $\mathbf{i}$ iff node $i$ is the parent of node $j[7]$. A node is a leaf node if it has no children, i.e., $q=\mathrm{d}-1$.

Lemma 1. In $S B T(s)$, any node $\mathrm{i}$ is at level $H(s, \mathrm{i})$, where $H(s, \mathrm{i})$ is the Hamming distance between $\mathrm{s}$ and $i$. The number of nodes at level $\mathrm{l}$ is $\left(\begin{array}{l}\boldsymbol{d} \\ \boldsymbol{l}\end{array}\right)$, and the height of the tree is $d$. The number of children nodes of any node $\mathrm{i}$ is $n_{c}=\mathrm{d}-1-q$, and $0 \leq n_{c} \leq d-H(s, \mathrm{i}) .[7]$

At the Eth broadcasting in Algorithm I, the root of the SBT is $P_{[k]}$. And because of the binaryreflected Gray code mapping, $P_{[k+1]}$ is adjacent to $P_{[k]}$ and therefore is at the first level of the SBT. However, with the SBT defined above, it is not guaranteed that $P_{[k+1]}$ is a leaf node, since by definition, $P_{[k+1]}$ is a leaf node in $\operatorname{SBT}\left(P_{[k]}\right)$ only if they differ by the high order $(d-1)$ st bit.

On the hypercube, if node $\mathrm{P}$ and node $P^{\prime}$ differ only by the $j$ th bit (and therefore they are directly linked), $0 \leq j<\mathrm{d}$, then we say $\mathbf{P}$ is the jth neighbor of $P^{\prime}$. Suppose $P_{[k]}$ and $P_{[k+1]}$ differ by the jth bit, we need to construct a SBT rooted at $P_{[k]}$ and with $P_{[k+1]}$ as a leaf node.

Definition $1 \mathrm{~A} S B T_{j}(s)$ in a d-cube is a $S B T$ rooted at node $\boldsymbol{s}$ and the jth neighbor node of $\boldsymbol{s}$ is a leaf node. (Note the $S B T(s)$ defined in [y] is therefore $S B T_{d-1}(s)$.)

Definition 2 The shufle operation on a node $i=\left(i_{d-1} i_{d-2} \cdots i_{1} i_{0}\right)$ is defined as $S(i)=$ $\left(\boldsymbol{i}_{d-2} \boldsymbol{i}_{d-3} \cdots \boldsymbol{i}_{1} i_{0} i_{d-1}\right)$. The inverse shufle operation on $\boldsymbol{i}$ is defined as $S^{-1}(\boldsymbol{i})=\left(\boldsymbol{i}_{0} \boldsymbol{i}_{d-1} \boldsymbol{i}_{d-2} \cdots \boldsymbol{i}_{1}\right)$. Moreover, $S^{k}(i)$ represents applying shufle operation $k$ times on $\mathrm{i}$, and $S^{-k}(i)$ represents applying inverse shuffle operation E times on $i$.

Definition 3 The shufle operation on a graph $G(V, \mathrm{E})$ is defined as $S(G)=G(S(V), S(E))$, where $S\left(V^{\prime}\right)=\{S(i) \mid \forall i \in V\}$ and $S(E)=\{(S(i), S(j)) \mid \forall(i, j) \in \mathrm{E})$. Similarly we define the inverse shuffle operation on a graph $S^{-1}(G)=G\left(S^{-1}(V), S^{-1}(E)\right)$.

Lemma 2 Shuffle and inverse shufle operations of a graph preserve the Hamming distance between nodes. The shufle operation $S^{k}$ maps every edge in dimension l to dimension $(l+k)$ mod $n$. The inverse shuffle operation $S^{-k}$ maps every edge in dimension $\mathbf{l}$ to dimension $(\boldsymbol{l}-\boldsymbol{k}) \bmod \boldsymbol{n} .[\gamma]$

Corollary 1 The topology of a graph remains unchanged under shuffle and inverse shufle operations. [7]

The next lemma shows that $S B T_{k}(s)$ can be obtained by applying shuffle and inverse shuffle operation on $S B T_{d-1}(s)$. 

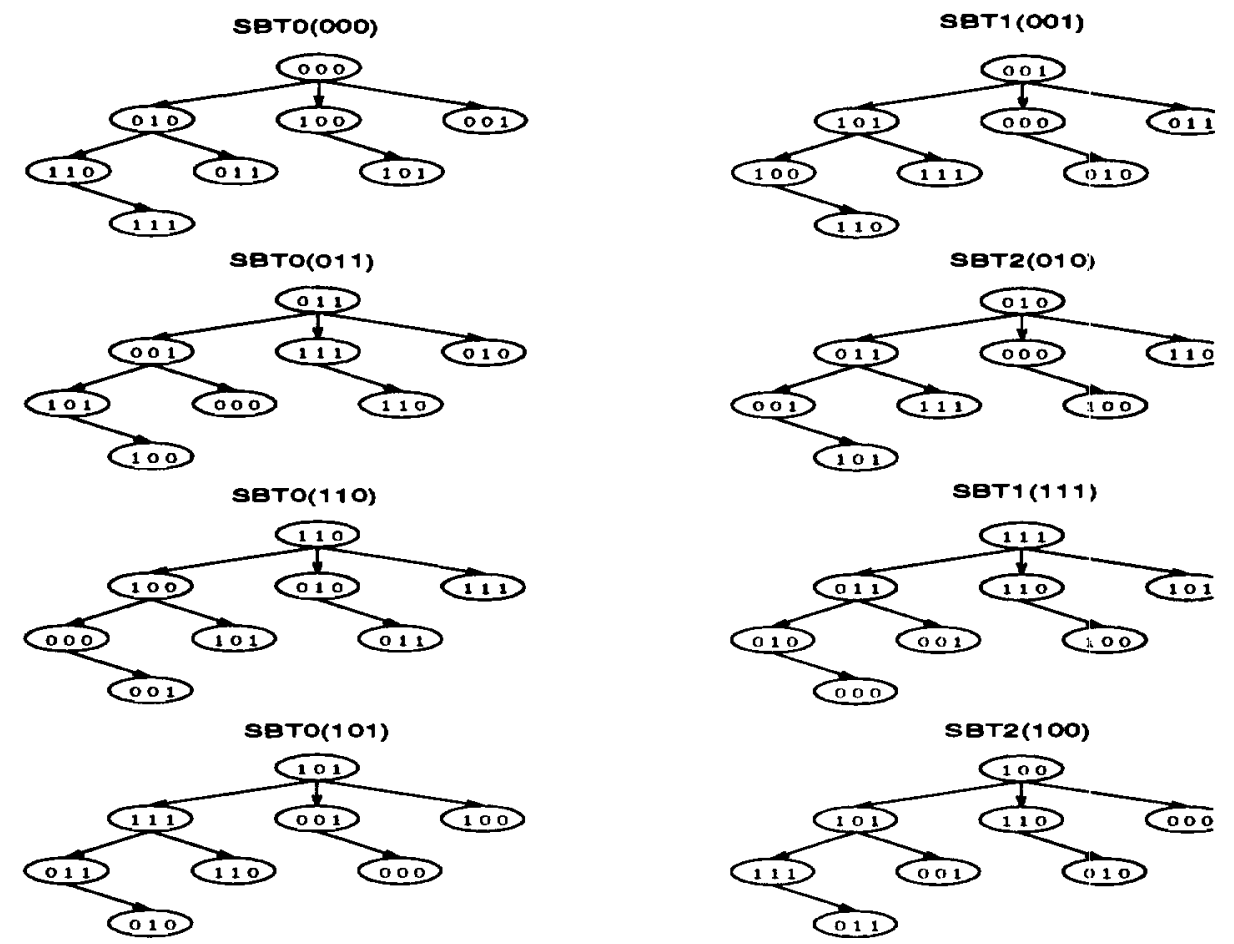

Figure 2: $\operatorname{SBT}(\operatorname{Gray}(3))$ is a family of $\mathbf{2}^{\mathbf{3}}=8$ SBT's. The root node of the [i+ 1]-st SBT is a leaf node of the $\mathrm{i}$-th SBT at the first level.

\section{Lemma 3}

$$
S B T_{k}(s)=S^{-(d-1-k)}\left[S B T_{d-1}\left(S^{d-1-k}(s)\right)\right]
$$

Proof: By Corollary $1, S^{-(d-1-k)}\left[S B T_{d-1}\left(S^{d-1-k}(s)\right)\right]$ is a SBT rooted at $S^{-(d-1-k)}\left[S^{d-1-k}(s)\right]=$ $s$. Let $i^{\prime}$ be the leaf node at the first level of $S B T_{d-1}\left(S^{d-1-k}(s)\right)$, then by definition, $i^{\prime}$ differs with $S^{d-1-k}(s)$ by the $(d-1)$ st bit. Let $i$ be the corresponding node of $i^{\prime}$ in $S^{-(d-1-k)}\left[S B T_{d-1}\left(S^{d-1-k}(s)\right)\right]$, by Lemma $2, \boldsymbol{i}$ and $s$ differ by the $(d-1)-(d-1-k)=k$ th bit. Therefore this SBT is $S B T_{k}(s)$. 口

The following theorem shows how to the construct $S B T_{k}(s)$ directly, by specifying the parent and children functions.

Theorem 1 In $S B T_{k}(s)$, let $q$ be such that $c_{q}=1$ and $c_{m}=0, \mathrm{Vm} \in M(c)=\{q+1$ mod $p, q+2$ $\bmod p, \cdots, k \bmod p)$, and let $q=k-d$ if $c=0$. Then

$$
\begin{aligned}
\operatorname{children}(i) & =\left\{\left(i_{d-1} i_{d-2} \cdots \bar{i}_{m} \cdots i_{0}\right)\right\}, \forall m \in M(c), \\
\operatorname{parent}(i) & = \begin{cases}\phi, & i=s \\
\left(i_{d-1} i_{d-2} \cdots \bar{i}_{q} \cdots i_{0}\right), & i \neq s\end{cases}
\end{aligned}
$$


Proof: Let $\boldsymbol{s}^{\prime}=S^{\boldsymbol{d - 1 - k}}(s), \boldsymbol{i}^{\prime}=S^{\boldsymbol{d - 1 - k}}(i), c^{\prime}=i^{\prime} \oplus \boldsymbol{s}^{\prime}$. Clearly $\boldsymbol{c}^{\prime}=S^{d-1-k}(\boldsymbol{c})$. Let $\boldsymbol{q}^{\prime}$ be such that $\boldsymbol{c}_{q^{\prime}}^{\prime}=1$ and $\boldsymbol{c}_{\boldsymbol{m}}^{\prime}=0, \forall m \in M\left(\boldsymbol{c}^{\prime}\right)=\left\{q^{\prime}+1, q^{\prime}+2, \ldots, d-1\right\}$. Then by definition, in $S B T_{d-1}\left(\boldsymbol{s}^{\prime}\right)$, the edges that connect $\boldsymbol{i}^{\prime}$ and its children nodes are those in dimension $d-1, d-2, \cdots, \boldsymbol{q}^{\prime}+1$. And the edge that connects $\boldsymbol{i}^{\prime}$ and its parent node is the one in dimension $\boldsymbol{q}^{\prime}$. Let $q=\boldsymbol{q}^{\prime}-(d-\mathbf{1}-k) \bmod$ $d$. From the definition of $q^{\prime}$ and the relation $c^{\prime}=S^{d-1-k}(c)$, it is easy to verify that $q$ satisfies the constraints given in the theorem. By Lemma $3, S B T_{k}(s)$ is obtained by inverse shuffle $S B T_{d-1}\left(s^{\prime}\right)$ $(d-1-k)$ times. Then by Lemma 2 , in $S B T_{k}(s)$, the edges that connect $i$ and its children nodes are those in dimension $d-1-\left(d-1-k \Rightarrow k, k-1 \bmod d, \cdots, \boldsymbol{q}^{\prime}+1-(\boldsymbol{d}-1--k) \bmod d=\boldsymbol{q}+1\right.$ $\bmod d$. And the edge that connects $i$ and its parent node is the one in dimension $q$.

The next theorem shows the structure of $\boldsymbol{S B} \boldsymbol{T}_{\boldsymbol{k}}(s)$.

Theorem 2 In $S B T_{k}(s)$, any node $i$ is at level $H(s, i)$, where $H(s, i)$ is the Hamming dislance between $\boldsymbol{s}$ and $\boldsymbol{i}$. The number of nodes at level 1 is $\left(\begin{array}{l}\boldsymbol{d} \\ \boldsymbol{l}\end{array}\right)$, and the height of the lree is $d$. The number of children nodes of any node $i$ is $(k-q) \bmod (d+1)$. The number of leaf nodes is $\mathbf{2}^{d-\mathbf{1}}$.

Proof: The first three statements follow directly Lemma 1, Lemma 2 and Corollary 1. By Theorem 1 , in $S B T_{k}(s)$ node $i$ is a leaf node if and only if the kth bit of $i\left(i_{k}\right)$ differs from the kth bit of $s\left(s_{i c}\right)$. For a given root $s$, there are $2^{d-1}$ nodes $i$ such that $i_{k} \neq s_{k}$. Therefore there are $2^{d-1}$ leaf nodes in $\operatorname{SBT}_{k}(\boldsymbol{s})$.

Next we give the construction of the p broadcasting trees used in Algorithm I.

Definition $4 S B T(\operatorname{Gray}(d))$ is a family of $p=2^{d} S B T$ 's:

$$
S B T(\operatorname{Gray}(d))=\left(S B T^{(1)}, S B T^{(2)}, \cdots, S B T^{\left(2^{d}\right)}\right),
$$

where $\operatorname{Gra} y(d)=\left(g_{1}^{d}, g_{2}^{d}, \cdots, g_{2^{d}-1}^{d}, g_{2^{d}}^{d}\right), S B T^{(i)}=S B T_{j(i)}\left(g_{i}^{d}\right)$, where $g_{i}^{d}$ and $\boldsymbol{g}_{[i+1]}^{d}$ differ by the $j($ i $)$-th bil.

As an example, consider $d=3$. We have

$$
\operatorname{Gray}(3)=(000,001,011,010,110,111,101,100)
$$

and Figure 2 shows the corresponding SBT's of $\operatorname{SBT}(\operatorname{Gray}(3))$.

The next theorem shows the structure of the set of trees, and will lead to the setup overhead complexity of the broadcasting algorithm.

Theorem. 3 In $S B T(G r a y(d))$, each node appears at lhe l-th level of () SBT's. Moreover, each node appears as a leaf node in $2^{d-1} S B T$ 's. 
Proof: By Theorem 2, in $S B T_{k}(\boldsymbol{s})$, any node $i$ is at level $H(s, \mathrm{i})$. For any fixed i, there are $\left(\begin{array}{l}d \\ l\end{array}\right) \boldsymbol{s}^{\prime}$ 's such that $H(s, \mathrm{i})=1$. And in the corresponding SBT's rooted at these nodes, $i$ is at level $?$.

To prove each node appears as a leaf node in $2^{d-1}$ SBT's, we use proof by induction on the hypercube dimension d. We use the first definition of binary-reflected Gray code. Induction hypothesis: In $S B T\left(g_{1}, g_{2}, . \cdot, g_{2^{d}}\right)$ or $S B T\left(g_{2^{d}}, \ldots, g_{2}, g_{1}\right)$, any node $i$ appears as leaf node in $2^{d-1}$ SBT's.

Base case: $d=1$. The proposition is obviously true.

Induction Step: Assume the proposition is true for dimension d, we show it is also true for dimension $\mathrm{d}+1$.

For $\operatorname{SBT}\left(g_{1}, g_{2}, \cdots, g_{2^{d}}\right)$, let

$$
\phi_{d}\left(i, g_{m}\right)= \begin{cases}1, & i \text { and } g_{m} \text { differ by the } j(m) \text { th bit } \\ 0, & \text { otherwise }\end{cases}
$$

where $g_{m}$ and $g_{[m+1]}$ differ by the $j(m)$ th bit.

And for $S B T\left(g_{2^{d}}, \cdots, g_{2}, g_{1}\right)$, let

$$
\phi_{d}^{\prime}\left(i, g_{m}\right)= \begin{cases}1, & i \text { and } g_{m} \text { differ by the } j^{\prime}(m) \text { th bit } \\ 0, & \text { otherwise. }\end{cases}
$$

where $g_{\boldsymbol{m}}$ and $\boldsymbol{g}_{[\boldsymbol{m}-1]}$ differ by the $\mathrm{j}^{1}(\mathrm{~m})$ th bit.

Then by the induction hypothesis,

$$
\sum_{m=1}^{2^{d}} \phi_{d}\left(i, g_{m}\right)=\sum_{m=2^{d}}^{1} \phi_{d}^{\prime}\left(i, g_{m}\right)=2^{d-1}
$$

For the d +1 dimension, we have $S B T\left(0 g_{1}, \cdots, 0 g_{2}^{d}, 1 g_{2}^{d}, \cdots, 1 g_{1}\right)$, for any node Oi, we have

$$
\begin{aligned}
& \sum_{m=1}^{2^{d+1}} \phi_{d+1}\left(0 i, g_{m}^{d+1}\right) \\
= & \sum_{m=1}^{2^{d}-1} \phi_{d}\left(i, g_{m}\right)+0+\sum_{m=2^{d}}^{2} \phi_{d}^{\prime}\left(i, g_{m}\right)+1 \\
= & \sum_{m=1}^{2^{d}} \phi_{d}\left(i, g_{m}\right)+\sum_{m=2^{d}}^{1} \phi_{d}^{\prime}\left(i, g_{m}\right)+1-\left(\phi_{d}\left(i, g_{2^{d}}\right)+\phi_{d}^{\prime}\left(i, g_{1}\right)\right) \\
= & 2^{d-1}+2^{d-1}+1-\left(\phi_{d}\left(i, g_{2^{d}}\right)+\phi_{d}^{\prime}\left(i, g_{1}\right)\right) .
\end{aligned}
$$

Suppose $g_{2^{d}}$ and $g_{1}$ differ by the jth bit. If $\phi_{d}\left(i, g_{2^{d}}\right)=1$ then the jth bits of $\boldsymbol{i}$ and $\boldsymbol{g}_{\mathbf{2}^{\boldsymbol{d}}}$ are different, so the jth bits of $i$ and $g_{1}$ are the same, and $\phi_{d}^{\prime}\left(i, g_{1}\right)=0$. Similarly, if $\phi_{d}^{\prime}\left(i, g_{1}\right)=1$, 
$\phi_{d}\left(i, g_{2^{d}}\right):=0$. Therefore $1-\left(\phi_{d}\left(i, g_{2^{d}}\right)+\phi_{d}^{\prime}\left(i, g_{1}\right)\right)=0$. Thus $\sum_{m=1}^{2^{d+1}} \phi_{d+1}\left(0 i, g_{n_{2}}^{d+1}\right)=2^{d}$.

$$
\begin{aligned}
& \sum_{m=1}^{2^{d+1}} \phi_{d+1}\left(1 i, g_{m}^{d+1}\right) \\
= & \sum_{m=1}^{2^{d}-1} \phi_{d}\left(i, g_{m}\right)+1+\sum_{m=2^{d}}^{2} \phi_{d}^{\prime}\left(i, g_{m}\right)+0 \\
= & \sum_{m=1}^{2^{d}} \phi_{d}\left(i, g_{m}\right)+\sum_{m=2^{d}}^{1} \phi_{d}^{\prime}\left(i, g_{m}\right)+1-\left(\phi_{d}\left(i, g_{2^{d}}\right)+\phi_{d}^{\prime}\left(i, g_{1}\right)\right) \\
= & 2^{d-1}+2^{d-1}+1-\left(\phi_{d}\left(i, g_{2^{d}}\right)+\phi_{d}^{\prime}\left(i, g_{1}\right)\right) \\
= & 2^{d}
\end{aligned}
$$

Therefore in $\boldsymbol{S B T}\left(\boldsymbol{g}_{1}^{d+1}, g_{2}^{d+1}, \cdots, g_{2^{d+1}}^{d+1}\right)$, any node appears as leaf node $\mathbf{2}^{d}$ times. Using the similar argument, we can show that in $\operatorname{SBT}\left(g_{2^{d+1}}^{d+1}, . ., g_{2}^{d+1}, g_{1}^{d+1}\right)$, any node appears as leaf node $2^{d}$ times.

The following corollary gives the setup overhead complexity of the broadcasting algorithm.

Corollary 2 In Algorithm I (row partitioning, with pivoting), when $S B T(G r a y(d)$ ) is used to broadcast the pivot rows, the total setup overhead of each processor is $\frac{1}{2} N t_{s}$.

Proof: Assume $N=n p$. There are totally $N$ one-to-all broadcasts, and $\operatorname{SBT}(\operatorname{Gray}(d))$ is used $\boldsymbol{n}=N / p$ times. By Theorem 3, in $S B T(G r a y(d))$, each node appears as leaf node $p / 2$ times. That is, for consecutive $p$ broadcastings, each processor incurs setup overhead of $\frac{1}{2} p t_{s}$. Therefore the total setup overhead for each processor in the $N$ broadcasts is $\frac{1}{2} p t_{s} \frac{N}{p}=\frac{1}{2} N t_{s}$. 


\section{Analysis of Algorithm I}

In this section we analyze the performance of the Algorithm I given in Section 3. The objective is to show the following:

1. A sufficient condition for full overlap of computation and communication.

2. The message queue length is bounded by a small number when full overlap is achieved.

3. Numerical results on the communication overhead of Algorithm I for partial or full overlap.

First consider the computation load of each processor at the Cth loop. The updating of each element not on the Cth column involves one multiplication and one subtraction, and the updating of each element on the Cth column involves one division. For simplicity, we assume the time to update each element of $\mathrm{A}$ is $\mathrm{f}$. For processor $P_{[k]}$, since it has already normalized the Cth pivot row and found the pivot element in the previous $(C-1)$ st loop, it needs to update the rest $(\mathrm{n}-1)$ rows it has using the $\mathrm{Cth}$ pivot row and pivot element. We call this computational task $\Pi_{1}$, and the corresponding computation time is $T_{1}$, where $T_{1}=(\mathrm{n}-1) \mathrm{Nf}$. For processor $P_{[k+1]}$, its computational task in the Cth loop can be split into two parts, $\Pi_{2}$ and $\Pi_{3} \cdot \Pi_{2}$ corresponds to updating the $\mathrm{C}+1$ st row of $\mathrm{A}$ using the Cth pivot row, normalizing this row and finding the pivot element of the $\boldsymbol{k}+1$ st pivot row. The time for updating is $\mathrm{Nf}$. We assume it takes another $\mathrm{Nf}$ to find the pivot element and normalize this row. Therefore the time for $\Pi_{2}$ is $T_{2}=2 \mathrm{Nf}$. After completing $\Pi_{2}$ processor $P_{[k+1]}$ immediately broadcasts the $(k+1)$ st pivot row. Then it resumes to update the other $(n-1)$ rows using the $C$ th pivot row, we call this task $\Pi_{3}$, and the corresponding time $T_{3}=T_{1}=(\mathrm{n}-1) \mathrm{Nf}$. For all the other processors, the computational task during the Cth loop is $\Pi_{\mathbf{4}}$, which corresponds to updating all the $\mathrm{n}$ rows using the $\boldsymbol{k}$ th pivoting row. The time for $\Pi_{4}$ is $T_{\mathbf{4}}=n N f$.

Therefore the computational load of processor $\mathbf{P}$ at the Cth loop of Algorithm I is given by

$$
T^{k}(P)= \begin{cases}T_{1}=(n-1) N f, & P=P_{[k]}, \\ T_{2}+T_{3}=(n+1) N f, & P=P_{[k+1]}, \\ T_{4}=n N f, & \text { otherwise }\end{cases}
$$

Note there is computational load imbalance among the processors in the $\boldsymbol{k}$ th loop. $P_{[k+1]}$ has the largest load and $P_{[k]}$ has the smallest load. By using the wrap-mapping to distribute the rows of A among processors, every processor becomes $P_{[k]}$ (and $P_{[k+1]}$ ) alternatively and thus the computation loads are balanced over the whole process of GJ inversion.

In order to explain and analyze our parallel GJ inversion algorithm, we introduce a task scheduling diagran, which is instrumental in our analysis of the performance of the proposed algorithm and allows us to conveniently formalize the notion of overlap communication and computation. A task scheduling diagram is a directed graph $G(V, \mathrm{E})$ consisting of a node set $\mathrm{V}$ and an arc set $\mathrm{E}$. 


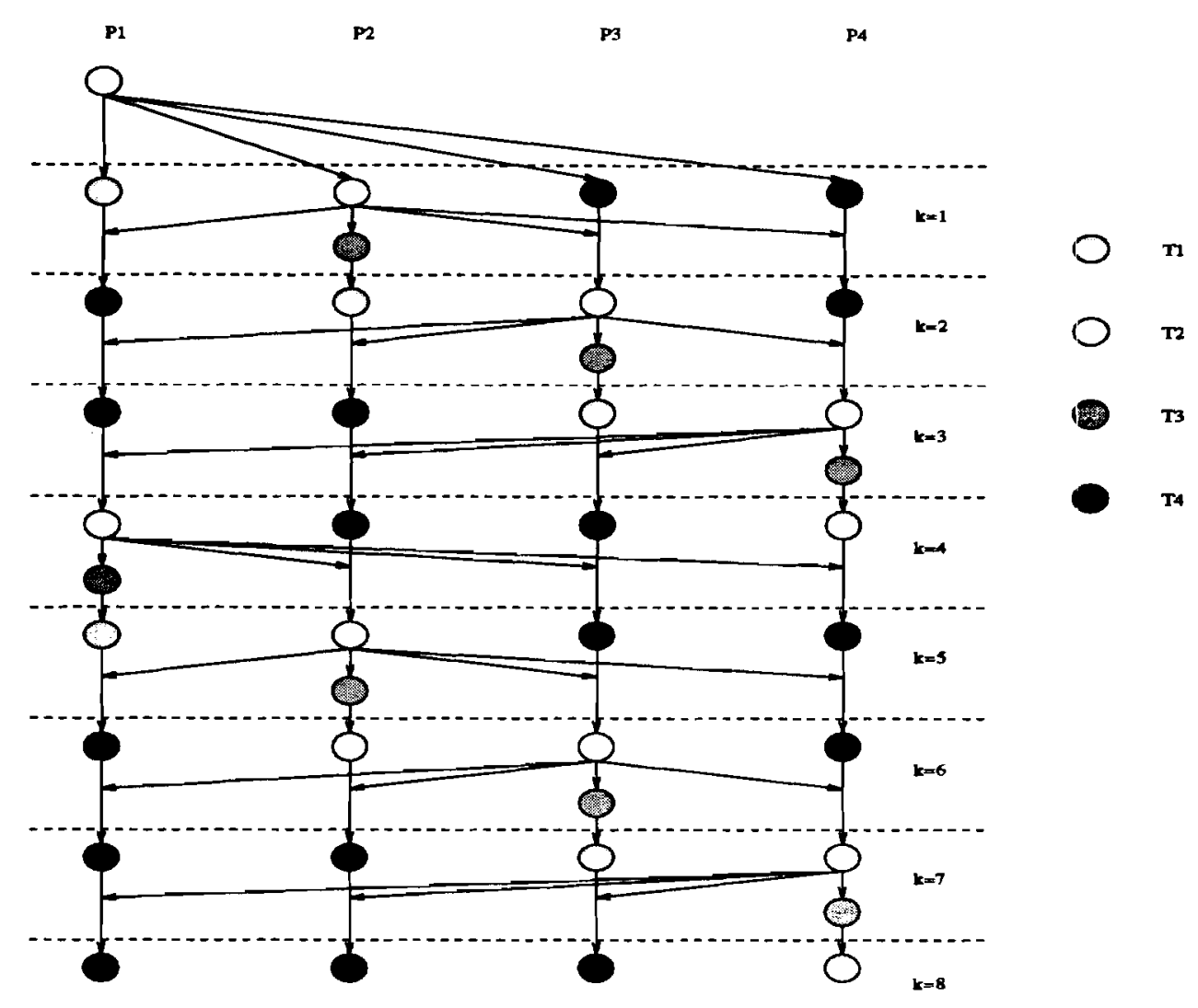

Figure 3: Task scheduling diagram for Algorithm I $(p=4$ and $N=8)$.

A node in $G$ represents one of the four computational tasks $\Pi_{1}, \Pi_{2}, \Pi_{3}$ and $\Pi_{4}$. An arc in $G$ represents precedence relations that exist between two computational tasks, subject to the condition that certain amount of time is allocated to each arc to account for the actual communication time between the two computational tasks represented by the two leaf nodes of the arc.

An example of a task scheduling diagram is given in Figure 3. It represents the parallel GJ inversion process of a $8 \times 8$ matrix using 4 processors. In general the task scheduling diagram $\mathrm{G}$ has the following properties:

Properfy 1: The nodes of G are ordered horizontally into p columns, each column \& corresponds to the updating operation sequence of processor $P_{l}$ during the parallel GJ inversion.

Properfy 2: Vertically $\mathrm{G}$ is constructed in $\mathrm{N}$ stages, each stage $\mathrm{k}$ corresponds to the concurrent updating operations of all the processors at the kth loop of the parallel GJ inversion.

Property 3: In the kth stage of $\mathrm{G}$, the node in column $[\mathrm{k}]$ is a $T_{1}$ node; in column $[\mathrm{k}+1]$ is a $T_{2}$ node followed $b y$ a $T_{3}$ node; and the nodes in all other columns are $T_{4}$ nodes.

Property 4: All nodes at the first stage of $G$ has in-degree 1. At stages other than the first, a $T_{1}$ or $T_{3}$ ncde has in-degree 1 , and a $T_{2}$ or $T_{4}$ node has in-degree 2 . 
Property 5: A $T_{2}$ node has out-degree $\mathrm{p}$ and the each of the other three type of nodes has out-degree 1, except in the last stage.

As can be seen in Figure 3, there are two kinds of arcs in C: "vertical" arcs and "non-vertical" arcs. They represent two kinds of precedence constraints and communication delays. A vertical arc is an arc connecting two adjacent nodes in the same column in C. The precedence constraint it represents is that the computation in the tail node must precede that of the head node, i.e., a processor can start the $(k+1)$ st loop only after it completes the $k$ th loop. The communication delay introduced by a vertical arc is the communication startup delay. As dliscussed earlier, a processor :may be interrupted by an incoming message and it may need to forward the message to other processors. This may introduce communication startup overhead and this overhead is represented by the vertical arc. A non-vertical arc is an arc from a $T_{2}$ node to a $T_{2}$ node or to a $T_{\mathbf{4}}$ node at the next stage in $\mathrm{G}$. The precedence constraint it represent is that the computation in the head node can not start before it receives the message from the tail node, i.e., a processor can not start the $(\mathrm{k}+1)$ st loop before it gets the $(\mathrm{k}+1)$ st pivot row from $P_{[k+1]}$. The communication delay introduced by a non-vertical arc is the time between the message is sent out and the message reaches the processor.

Next we give an analytical model for the performance of Algorithm I. First some notations are listed as following.

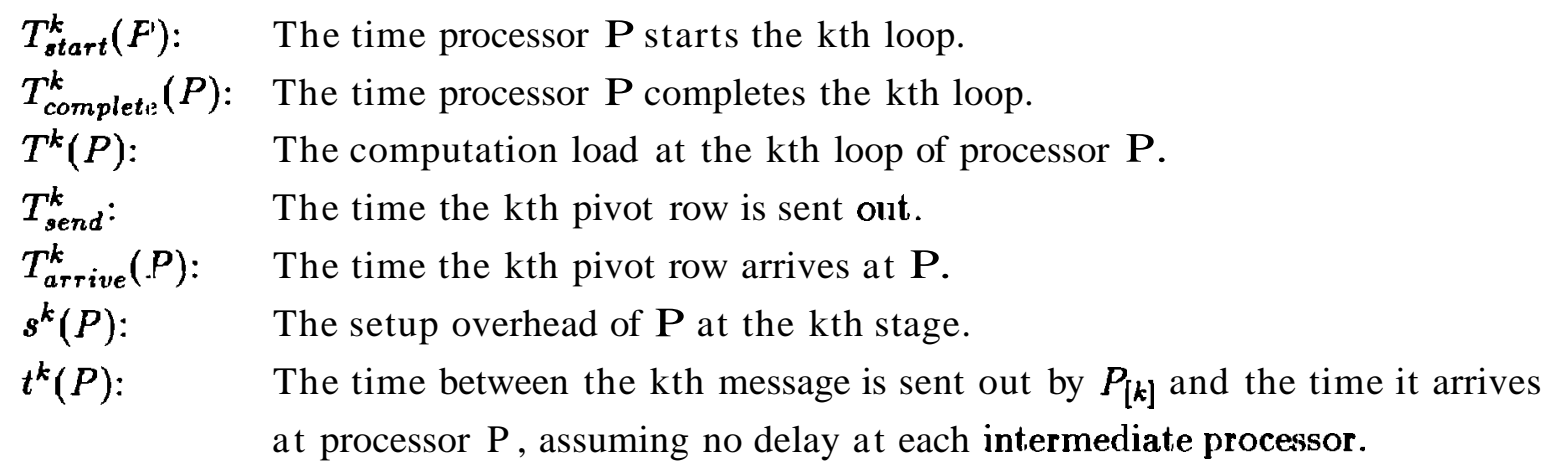

At stage k in $G$, a $T_{2}$ or a $T_{4}$ node has two incoming arcs, the delay introduced by the vertical arc is $\boldsymbol{s}^{k}(P)$ and the delay introduced by the non-vertical arc is $t^{k}(P)$. From Section 4 we know that $s^{k}(P)$ is either 0 or $\mathrm{t}$, depending on whether or not $\mathrm{P}$ is a leaf node in the kth broadcasting tree. Let $H\left(P_{[k]}\right.$ P $)$ be the Hamming distance between the physical addresses of $P_{[k]}$ and $\mathrm{P}$. Then the message will take $H\left(P_{[k]}, \mathrm{P}\right)$ hops to reach $\mathrm{P}$ from $P_{[k]}$. Therefore $t^{k}(P)=H\left(P_{[k]}, P\right)\left(t_{s}+t_{w} N\right)$. A $T_{1}$ node has one vertical incoming arc, which introduces no delay, i.e., $s^{k}(P)=0$. A $T_{3}$ node has one vertical incoming arc, which introduces delay $\boldsymbol{s}^{\boldsymbol{k}}(P)=\boldsymbol{t}_{\boldsymbol{s}}$.

Figure 4 illustrates the relationship among the parameters defined above, which can be formally stated by the following lemma.

Lemma 4 We have Ihe following recurrent relationships: 


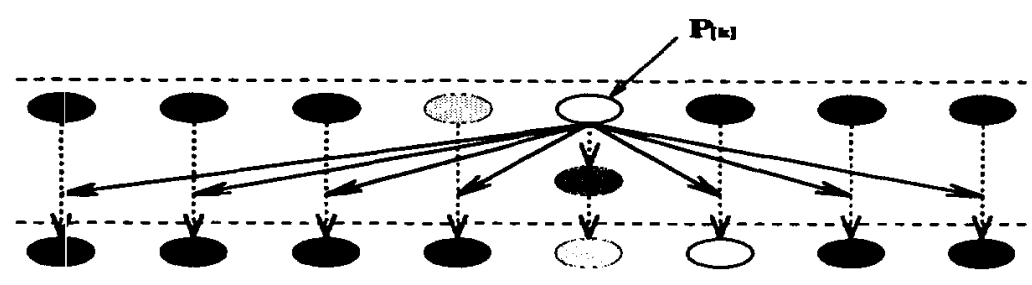

$(k-1)$ st iteration

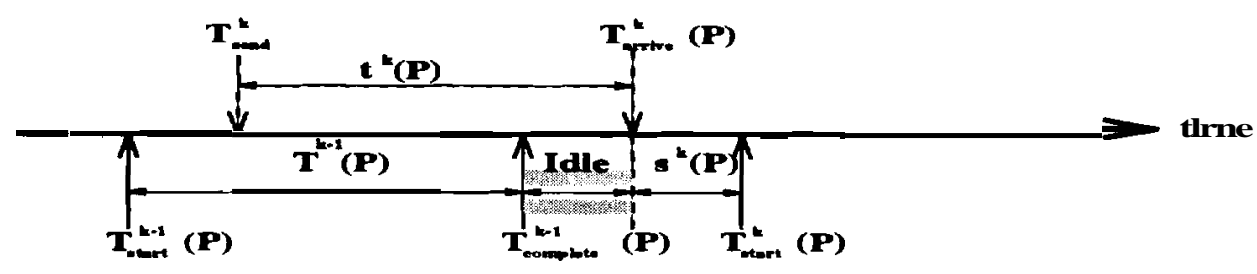

Idle time $>0$

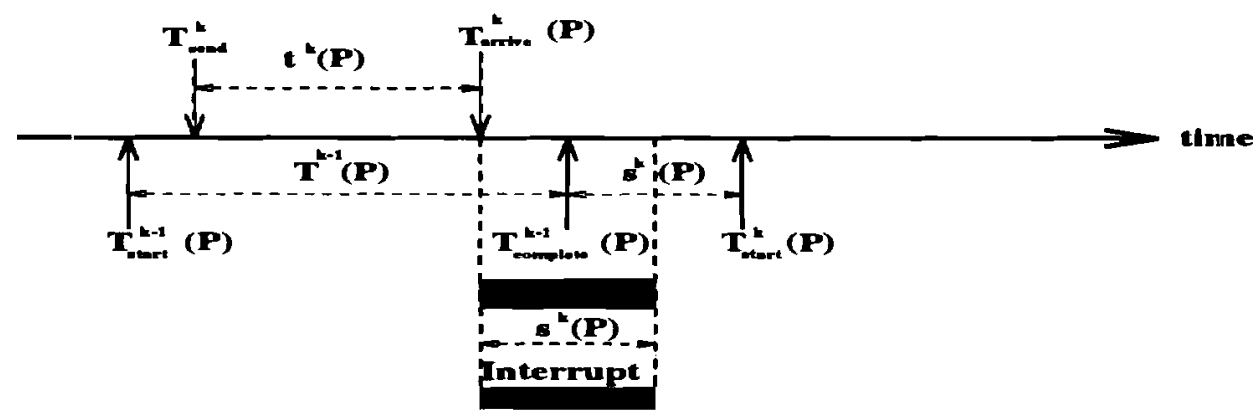

Idle time $=0$

Figure 4: Illustration of the relationship among the parameters defined above.

$$
\begin{gathered}
T_{\text {start }}^{k}(P)= \begin{cases}\max \left\{T_{\text {complete }}^{k-1}(P), T_{\text {arrive }}^{k}(P)\right\}+s^{k}(P), & P \neq P_{[k]} \\
T_{\text {complete }}^{k-1}(P)+s^{k}(P), & P=P_{[k]}\end{cases} \\
T_{\text {complete }}^{k}(P)=T_{\text {start }}^{k}(P)+T^{k}(P) . \\
T_{\text {send }}^{k}=T_{\text {start }}^{k-1}\left(P_{[k]}\right)+T_{2} .
\end{gathered}
$$

Proof: 'These relationships are obvious from the definition of $T_{\text {start }}^{k}(P), T_{\text {complete }}^{k}(P)$ and $T_{\text {send }}^{k}$, and the task scheduling graph, as illustrated in Figure 4.

The next theorem states that a message can not be delayed at an intermediate processor during the broadcast process in Algorithm I. 
Theorem.4 In Algorithm I, when the SBT, broadcast algorithm is used, then

$$
T_{\text {arrive }}^{k}(P)=T_{\text {send }}^{k}+t^{k}(P) \text {. }
$$

Proof: We need to show that whenever a message arrives at an intermediate processor, the processor can forward this message immediately without delay. A delay is incurred if when the message arrives at the processor, the processor is setting up the communication channel for a previous nnessage. Then only after the processor finishes setting up the communication channel for the previous message can it start forward this message. In the worst case the message can be delayed for: up to time t,.

We have the following two simple observations:

(1) If $T_{\text {arrive }}^{k}(P)-T_{\text {arrive }}^{k-1}(P)>t_{s}$, then message $k$ will not be delayed at processor $\mathrm{P}$, because when message $k$ arrives at $\mathrm{P}, \mathrm{P}$ has already finished setting up communication clhannel for message $k-1$, and therefore can forward message $\mathrm{k}$ immediately.

(2) If $P$ does not forward message $\mathrm{k}-1$, i.e., $\mathbf{P}$ is a leaf node of the $(\mathrm{k}-1)$ st SBT, then as long as $T_{a r r i v e}^{k}(P)-T_{a r r i v e}^{k-1}(P)>0$, message $\mathrm{k}$ will not be delayed at processor $\mathrm{P}$, since $\mathrm{P}$ can immediately forward message $k$.

Assume $H\left(P_{[k-1]}, P\right)=\mathrm{m}$, since $H\left(P_{[k]}, P_{[k-1]}\right)=1$, we have either $H\left(P_{[k]}, P\right)=\mathrm{m}+1$ or $H\left(P_{[k]}, P\right)=\mathrm{m}-1$. Suppose $P_{[k]}$ and $P_{[k-1]}$ differ by the jth bit, if $P$ and $P_{[k-1]}$ have the same jth bit, then $\mathrm{P}$ and $P_{[k]}$ have different jth bits, and $H\left(P_{[k]}, \mathrm{P}\right)=\mathrm{m}+1$; otherwise $\mathrm{P}$ and $P_{[k-1]}$ have different $\mathrm{jth}$ bits, then $\mathrm{P}$ and $P_{[k]}$ have the same jth bit, and $H\left(P_{[k]}, \mathrm{P}\right)=\mathrm{m}-1$.

Notice that

$$
\begin{aligned}
& T_{\text {send }}^{k}-T_{\text {send }}^{k-1} \\
= & {\left[T_{\text {start }}^{k-1}\left(P_{[k]}\right)+T_{2}\right]-T_{\text {send }}^{k-1} } \\
\geq & \left(T_{\text {send }}^{k-1}+t_{s}+t_{w} N+T_{2}\right)-T_{\text {send }}^{k-1} \\
= & \left(t_{s}+t_{w} N\right)+T_{2} .
\end{aligned}
$$

The proof is by induction on $k$. For $\mathrm{k}=1$ obviously the message will not be delayed at any processor since there is no other previous message. Assume message $(\mathrm{k}-1)$ is not delayed at any processor $\boldsymbol{P}$, i.e.,

$$
T_{\text {arrive }}^{k-1}(\mathrm{P})=T_{\text {send }}^{k-1}+t^{k-1}(P)=T_{s e n d}^{k-1}+m\left(t_{s}+t_{w} N\right) .
$$

We need to show message $\mathrm{k}$ also can not be delayed. We consider two cases:

(1) $H\left(P_{[k]}, P\right)=m+1$. Then $T_{\text {arrive }}^{k}(P)-T_{\text {arrive }}^{k-1}(P) \geq T_{\text {send }}^{k}+(m+1)\left(t_{s}+t_{w} N\right)-T_{\text {arrive }}^{k-1}(P)=$ $2\left(t_{s}+t_{w} N\right)+T_{2}>\mathrm{t}$. . Therefore message $\mathrm{k}$ will not be delayed at processor $\mathrm{P}$.

(2) $H\left(P_{[k]}^{2}, P\right)=\mathrm{m}-1$. Then $T_{\text {arrive }}^{k}(P)-T_{\text {arrive }}^{k-1}(P) \geq T_{\text {send }}^{k}+(m-1)\left(t_{s}+t_{w} N\right)-T_{\text {arrive }}^{k-1}(P)=$ $T_{2}>0$. Since $\mathrm{P}$ and $P_{[k-1]}$ have different jth bits, $\mathrm{P}$ is a leaf node in the (k-1jst SBT, therefore $\mathbf{P}$ does not forward message $(\mathrm{k}-1)$. So message $\mathrm{k}$ will not be delayed at processor $\mathbf{P}$. 
Let $T_{\text {cэmp }}(P)$ be the total computation load of processor $P$, and $T_{\text {comm }}(P)$ be the total communication overhead of processor $\boldsymbol{P}$, then

$$
\begin{aligned}
T_{\text {comp }}(P) & =\sum_{k=1}^{N} T^{k}(P) \\
T_{\text {comm }}(P) & =T_{\text {complete }}^{N}(P)-T_{\text {comp }}(P) \\
& =T_{\text {complete }}^{N}(P)-\sum_{k=1}^{N} T^{k}(P) .
\end{aligned}
$$

It can be seen in Figure 4 that at the $(k-1)$ st iteration, if $T_{\text {arrive }}^{k}(P)>T_{\text {complete }}^{k-1}(P)$, then processor $P$ will be idle for a time $t_{i d l e}^{k-1}(P)=T_{\text {arrive }}^{k}(P)-T_{\text {complete }}^{k-1}(P)$; otherwise $t_{\text {idle }}^{k-1}(P)=0$. If $\boldsymbol{t}_{\boldsymbol{i d l e}}^{k}(P)=\mathbf{0}$ for all $2 \leq k \leq N$, then full overlap is achieved at $\boldsymbol{P}$. Now we can give a formal definition of full overlap of communication and computation.

Definition 5 We say that the communication is fully overlapped by computation for processor $\boldsymbol{P}$ in Algorithm I if

$$
T_{\text {complete }}^{k-1}(P) \geq T_{\text {send }}^{k}+t^{k}(P), \quad 2 \leq k \leq N
$$

or equivalently

$$
T_{\text {start }}^{k}(P)=T_{\text {complete }}^{k-1}(P)+s^{k}(P), \quad 2 \leq k \leq N .
$$

That is, the kth pivot row has already arrived at processor $\boldsymbol{P}$ when $\boldsymbol{P}$ completes computation of the $k-1$ st stage of the parallel GJ inversion algorithm.

At the 'beginning of the algorithm, i.e., $k=1$, all the processors except $P_{\mathbf{1}}$ are idle until the first pivot row arrives, as illustrated in Figure $\mathbf{3}$. Let $\boldsymbol{d}_{0}(P)$ be the initial idle time of ; processor $\boldsymbol{P}$, Since $P_{\mathbf{1}}$ first normalizes the first row of A and finds the pivot element, which takes time $N f$, before it sends it out, then $d_{0}(P)=H\left(P_{1}, P\right)\left(t_{s}+t_{w} N\right)+N$ f

Since our objective is to let the message arrive at all the processors before it is needed, another parameter of interest is the number of messages in the input buffer of a processor at a particular time.

Definition 6 Let $\boldsymbol{Q}^{k}(P)$ be the message queue length of processor $\boldsymbol{P}$ at stage $\boldsymbol{k}$, then $Q^{k}(P)=l$, if

$$
T_{\text {send }}^{k+l}+t^{k+l}(P) \leq T_{\text {complete }}^{k}(P)<T_{s e h d}^{k+1}+t^{k+l+1}(P)
$$

That is, after processor $\boldsymbol{P}$ completes stage $\boldsymbol{k}$ and is to start stage $k+1$, messages for stage $\boldsymbol{k}+$ $1, \boldsymbol{k}+2, \ldots, k+\mathbf{l}$ have already arrived at $P$.

The next theorem gives a sufficient condition for full overlap communication and computation in Algorithm I. 
Theorem 5 When $\mathrm{N} \geq N_{0}$, full overlap ofcommunication and computation can be achieved, where $N_{\mathbf{0}}$ is the positive root of the quadratic equation

$$
\left(\frac{N}{p}-3\right) N f=\frac{1}{2} p t_{s}+2\left(t_{s}+t_{w} N\right) \log p
$$

Proof: We rewrite the quadratic equation in the standard form:

$$
\left(\frac{f}{p}\right) N^{2}-\left(3 f+2 t_{w} \log p\right) \mathrm{N}-\left(\frac{1}{\hbar} p+2 \log p\right) t_{s}=0 .
$$

Obviously this equation has one positive root and one negative root. Let the positive root be $N_{0}$. When $\mathrm{N} \geqq N_{0}$, we have

$$
\left(\frac{\mathrm{N}}{\mathrm{P}}-3\right) N f \geq \frac{1}{2} p t_{s}+2\left(t_{s}+t_{w} N\right) \log p .
$$

Let $\Delta T^{k}(P)=T_{\text {complete }}^{k-1}(\mathrm{P})-T_{\text {send }}^{k}-t^{k}(P)$, we will show that $\Delta T^{k}(P) \geq 0$ for $2 \leq \mathrm{k} \leq \mathrm{N}$ and $\mathrm{P} \in\left\{P_{1}, P_{2}, \cdots, P_{p}\right\}-P_{k}$. The proof is by induction.

(1) Base case: $\mathrm{k}=2$,

$$
\begin{aligned}
\Delta T^{2}(P) & =T_{\text {complete }}^{1}(P)-T_{\text {send }}^{2}-t^{2}(P) \\
& =\left[T_{\text {start }}^{1}(P)+T^{1}(P)\right]-\left[T_{\text {start }}^{1}\left(P_{2}\right)+T_{2}\right]-t^{2}(P) \\
& =\left(d_{0}(P)+T^{1}(P)\right)-\left(d_{0}\left(P_{2}\right)+T_{2}\right)-t^{2}(P) \\
& =\left(T^{1}(P)-T_{2}\right)-\left(d_{0}\left(P_{2}\right)-d_{0}(P)\right)-t^{2}(P) .
\end{aligned}
$$

where $T^{1}(D)-T_{2}$

$$
\begin{gathered}
= \begin{cases}T_{1}-T 2=(n-3) N f, & P=P_{1} \\
T_{4}-T 2=n N f-2 N f=(n-2) N f, & P \neq P_{1} .\end{cases} \\
d_{0}\left(P_{2}\right)-d_{0}(P) \leq\left(t_{s}+t_{w} N\right) \log p . \\
t^{2}(P) \leq\left(t_{s}+t_{w} N\right) \log p .
\end{gathered}
$$

Therefore

$$
\begin{aligned}
\Delta T^{2}(P) & \geq(\mathrm{n}-3) \mathrm{Nf}-2\left(t_{s}+t_{w} N\right) \log p-N f \\
& =\left(\frac{\mathrm{N}}{\mathrm{P}}-3\right) \mathrm{Nf}-2\left(t_{s}+t_{w} N\right) \log \mathrm{p} \\
& >0
\end{aligned}
$$

when $\mathrm{N} \geq N_{0}$.

(2) Induction step: Assume the proposition is true for $2 \leq l \leq k$. We prove it is also true for $l=k+1$. 
By the induction hypothesis, $T_{\text {start }}^{l}(P)=T_{\text {complete }}^{l-1}(P)+s^{l}(P)$, for $2 \leq l \leq \mathrm{k}$. Therefore

$$
\begin{aligned}
& \Delta T^{k+1}(P) \\
= & T_{\text {complete }}^{k}(P)-T_{\text {send }}^{k+1}-t^{k+1}(P) \\
= & {\left[T_{s t a r t}^{k}(P)+T^{k}(P)\right]-\left[T_{s t a r t}^{k}\left(P_{[k+1]}\right)+T_{2}\right]-t^{k+1}(P) } \\
= & {\left[d_{0}(P)+\sum_{i=1}^{k}\left(T^{i}(P)+s^{i}(P)\right)\right]-\left[d_{0}\left(P_{[k+1]}\right)+\sum_{i=1}^{k}\left(T^{i}\left(P_{[k+1]}\right)+s^{i}\left(P_{[k+1]}\right)\right)-T^{k}\left(P_{[k+1]}\right)+T_{2}\right] } \\
& -t^{k+1}(P) \\
= & \sum_{i=1}^{k}\left[T^{i}(P)-T^{i}\left(P_{[k+1]}\right)\right]+\left(T_{[k+1]}^{k}-T_{2}\right)-\left[d_{0}\left(P_{[k+1]}\right)-d_{0}(P)\right] \\
& -\left[\sum_{i=1}^{k} s^{i}\left(P_{[k+1]}\right)-\sum_{i=1}^{k} s^{i}(P)\right]-t^{k+1}(P) \\
> & \sum_{i=1}^{k}\left[T^{i}(P)-T^{i}\left(P_{[k+1]}\right)\right]+\left(T_{2}+T_{3}-T_{2}\right)-\left(t_{s}+t_{w} N\right) \log p-\frac{1}{2} p t_{s}-\left(t_{s}+t_{w} N\right) \log p \\
= & -N j^{e}+(n-l) N f-\frac{1}{2} p t_{s}-2\left(t_{s}+t_{w} N\right) \log p \\
= & \left(\frac{N}{p}-2\right) N f-\frac{1}{2} p t_{s}-2\left(t_{s}+t_{w} N\right) \log p \\
\geq & 0,
\end{aligned}
$$

when $\mathbf{N} \geq N_{0}$. Here we used the fact that if let $r=\left\lceil\frac{k}{p}\right\rceil$, then

$$
\sum_{i=1}^{k} T^{i}(P)= \begin{cases}\left(T_{1}+T 2+T_{3}\right) r+T_{4}(k-2 r), & P \in\left\{P_{1}, P 2, \cdots, P_{[k]}\right\} \\ \left(T_{1}+T 2+T_{3}\right) r-T_{1}+T_{4}(k-2 r+1), & P=P_{[k+1]} \\ \left(T_{1}+T 2+T_{3}\right)(r-1)+T_{4}(k-2 r+2), & P \in\left\{P_{[k+2]}, \cdots, P_{p}\right\}\end{cases}
$$

And therefore

$$
\sum_{i=1}^{k}\left[T^{i}(P)-T^{i}\left(P_{[k+1]}\right)\right]= \begin{cases}T_{1}-T_{4}=-N f, & P \in\left\{P_{1}, P 2, \cdots, P_{[k]}\right\}, \\ T_{4}-\left(T_{2}+T_{3}\right)=-N f, & P \in\left\{P_{[k+2]}, \cdots, P_{p}\right\} .\end{cases}
$$

We also used the fact that

$$
\begin{aligned}
& \sum_{i=1}^{k} s^{i}\left(P_{[k+1]}\right)-\sum_{i=1}^{k} s^{i}(P) \\
= & \left\lfloor\frac{k}{p}\right\rfloor\left(\frac{1}{2} p t_{s}-\frac{1}{2} p t_{s}\right)+\sum_{i=1}^{[k]} s^{i}\left(P_{[k+1]}\right)-\sum_{i=1}^{[k]} s^{i}(P) \\
\leq & \frac{1}{2} p t_{s} .
\end{aligned}
$$

The next theorem states that the message queue length at each processor is lbounded by 2 . 
Theorem 6 When $\mathrm{N} \geq N_{0}$, the message queue length is at most 2 , i.e., $Q^{k}(P) \leq 2$.

Proof: By contradiction. Otherwise there exist $k$ and $\mathrm{P}$ such that $Q^{k}(P)=l \geq 3$. Since $\mathrm{N} \geq N_{0}$, by Theorem 5 , communication is fully overlapped by computation. By the definition of the queue length $Q^{k}(P)$, we have

$$
\begin{aligned}
T_{\text {complete }}^{k}(P) & \geq T_{\text {send }}^{k+l}+t^{k+l}(P) \\
& =T_{\text {start }}^{k+l-1}\left(P_{[k+l]}\right)+T_{2}+t^{k+l}(P) \\
& =T_{\text {complete }}^{k+l-2}\left(P_{[k+l]}\right)+\boldsymbol{s}^{k+l-1}\left(P_{[k+l]}\right)+T_{2}+t^{k+l}(P) .
\end{aligned}
$$

Or

$$
\begin{gathered}
d_{0}(P)+\sum_{i=1}^{k}\left[T^{i}(P)+s^{i}(P)\right] \geq d_{0}\left(P_{[k+l]}\right)+\sum_{i=1}^{k+l-2}\left[T^{i}\left(P_{[k+l]}\right)+s^{i}\left(P_{[k+l]}\right)\right] \\
+s^{k+l-1}\left(P_{[k+l]}\right)+T_{2}+t^{k+l}(P)
\end{gathered}
$$

$$
\begin{aligned}
\sum_{i=1}^{k+l-2} T^{i}\left(F_{[k+l]}\right)-\sum_{i=1}^{k} T^{i}(P)+T_{2} & \leq\left(d_{0}(P)-d_{0}\left(P_{[k+l]}\right)\right)-t^{k+l}(P)-\sum_{i=1}^{k+l-1} s^{i}\left(P_{[k+l]}\right)+\sum_{i=1}^{k} s^{i}(P) \\
& \leq\left(t_{s}+t_{w} N\right) \log p+\frac{1}{2} t_{s}
\end{aligned}
$$

But when $d \geq 3$,

$$
\begin{aligned}
\sum_{i=1}^{k+l-2} T^{k}\left(P_{[k+l]}\right)-\sum_{i=1}^{k} T^{k}(P)+T_{2} & \geq T_{1}+T_{2} \\
& =\left(\frac{N}{p}+1\right) N f \\
& >\left(\frac{N}{p}-3\right) N f \\
& \geq 2\left(t_{s}+t_{w} N\right) \log p+\frac{1}{2} p t_{s} \\
& >\left(t_{s}+t_{w} N\right) \log p+\frac{1}{2} p t_{s} .
\end{aligned}
$$

(1) and (2) contradicts each other. Therefore $Q^{k}(P) \leq 2$.

Corollary 3 When $\mathrm{N} \geq N_{0}$, the communication overhead of processor $P$ in Algorithm I is $d_{0}(P)+$ $\frac{N}{2} t_{s}$.

Proof: At the beginning of the algorithm $(\mathrm{k}=1)$, the processors other than $P_{1}$ must wait to get the first pivot row from $P_{\mathbf{1}}$, since no computation can be done for these processors before getting the first pivot row. Thus a communication overhead (initial idle time) of $\boldsymbol{d}_{0}(P)$ is incurred. After that, since $\mathrm{N} \geq N_{0}$ by Theorem 5 , the data transmission is fully overlapped by computation for 


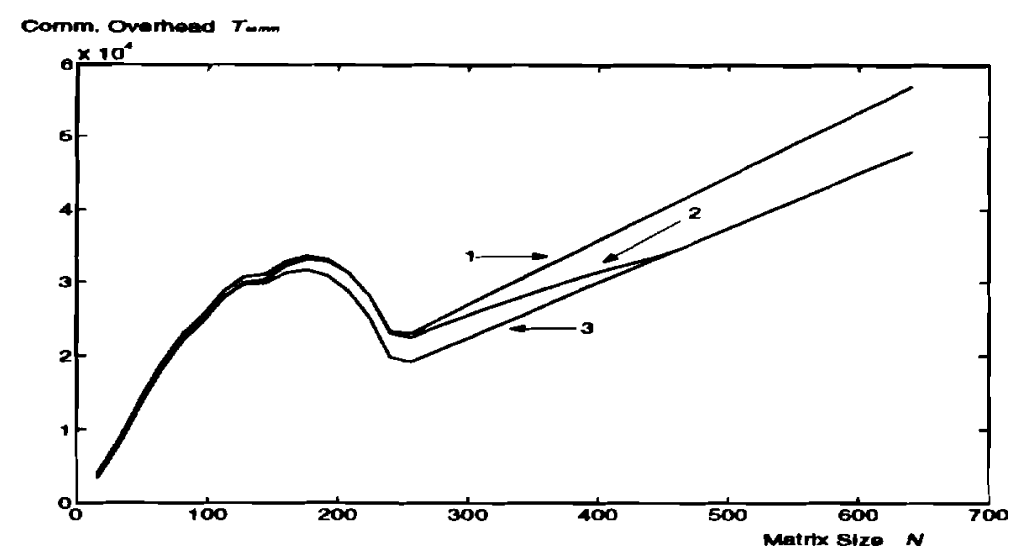

Figure 5: Communication overheads of Algorithm I (Number of processors $p=16$ ).

all the processors during the rest of the updating process (for $2 \leq k \leq \mathrm{N}$ ). Therefore the total idle time for processor $\mathbf{P}$ in Algorithm $\mathrm{I}$ is $\boldsymbol{d}_{\mathbf{0}}(P)$. By Corollary 2, the total setup overhead of each processor in Algorithm I is $\frac{N}{2} t_{s}$. So the total communication overhead is $d_{0}(P)+\frac{N}{2} t_{s}$.

Next w'e give some simulation results on the parallel overheads of Algoritlhm I, obtained by numerically evaluating the recurrent relationships given in Lemma 4 . The time is scaled to $f$, i.e., let $\boldsymbol{f}=1$. We use the machine parameter $\mathrm{t},=\mathbf{1 5 0}$ and $\boldsymbol{t}_{\boldsymbol{w}}=3$. These parameters are close to that of the nCUBE 2 machine [28]. Since the communication overheads may be different for different processors, the data depicted in the figure are the largest overheads among the processors. Figure 5 depicts the communication overhead $T_{\text {comm }} v$ s the matrix size $\mathrm{N}$ when the machine size $\mathrm{p}=\mathbf{1 6}$. Curve $\mathbf{1}$ is the total communication overheads; curve $\mathbf{2}$ is the total communication overheads minus the initial delays; for the case of curve 3, we let each processor initially holcls the first row of A, such that all processors can start without the initial delay. It can be seen that when there is initial delay, when $\mathrm{N}>\mathbf{2 6 0}$ the communication is "almost" overlapped by computation, but not completely until $\mathrm{N}>460$. When there is no initial delay, the communication is completely overlapped by computation when $\mathrm{N}>260$.

Figure 6 shows the communication overheads with different machine sizes. The initial delays are subtracted so that it is easy to see where full overlap is achieved. We can see that when full overlap is achieved, the communication overhead is independent of the machine size (if the initial delay is not considered), and is linearly increasing with the problem size (i.e., $T_{\text {comm }}=\frac{1}{2} N t_{s}$ ). 


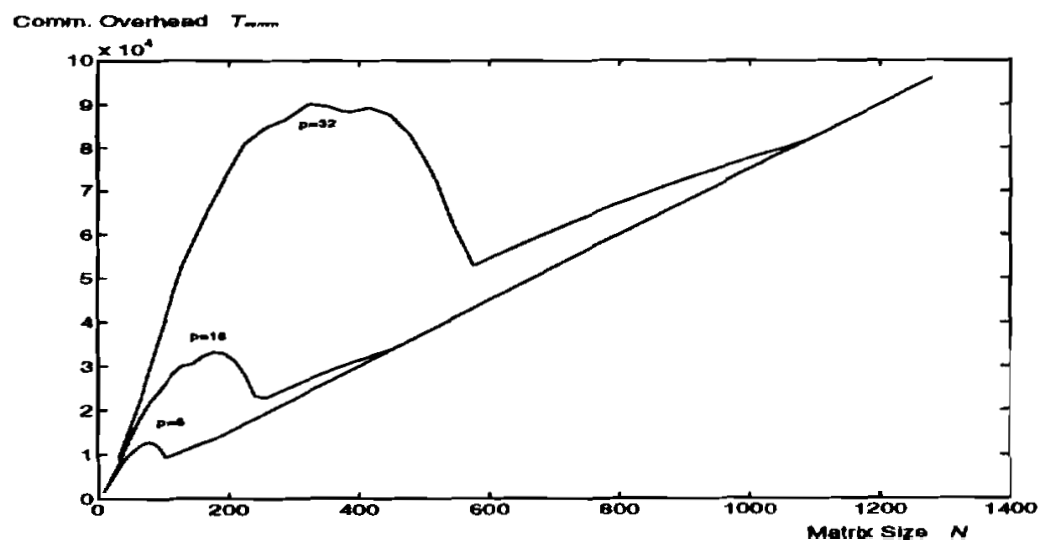

Figure 6: Communication overheads of Algorithm I with different machine sizes. 


\section{Algorithm II: Submatrix Partitioning, Without Pivoting}

In this section, we apply the idea of overlap communication and computation to the parallel GJ matrix inversion algorithm using submatrix partitioning. The main advantages of the submatrix partitioning are shorter message length, i.e., the message is of length $\frac{N}{\sqrt{p}}$ instead of $\mathrm{N}$; and shorter communication propagation delay, i.e., each broadcasting tree is of height $\frac{d}{2}$ instead of $d$. However, the disadvantage is that the searching of pivot element can not be done by a single processor. Instead $\sqrt{\bar{p}}$ processors individually find their local pivot element and compare with each other to find the final pivot element through recursive doubling. As a result, at every iteration there is a synchronization barrier among all the processors which makes it difficult to overlap communication and computation. In this section, we study the algorithm without pivoting.

Let $\mathrm{p}=2^{d}$ be the number of processors in a $\mathrm{d}$-cube. These processors are configured as a two dimerisional array of processors. Let $\mathrm{p}$, and $p_{c}$ be the number of processor rows and columns respectively, where $\mathrm{p},=p_{c}=\sqrt{p}=2^{d / 2}$ when $\mathrm{d}$ is even, and $p_{r}=\sqrt{2 p}=2^{(d+1) / 2}, p_{c}=\sqrt{p / 2}=$ $2^{(d-1) / 2}$ when $d$ is odd. The physical address of each processor is determined by the 2-d Gray code mapping. Let $\mathrm{d},=\log \mathrm{p}, \boldsymbol{d}_{c}=\log \mathrm{p}$, and $\mathrm{d}=\max \left(\boldsymbol{d}_{r}, \boldsymbol{d}_{c}\right)$. The d-bit Gray code is $\boldsymbol{G r a y}(\boldsymbol{d})=$ $\left(g_{1}^{d}, g_{2}^{d}, \cdots, g_{2^{d}}^{d}\right)$. We use notation $P_{i j}$ to denote the processor with coordinates $(\mathrm{i}, \mathrm{j})$ in the processor array, where $1 \leq \mathrm{i} \leq \mathrm{p}$, and $1 \leq \mathrm{j} \leq p_{c}$. Then the physical address of $P_{i j}$ is $g_{i}^{d} g_{j}^{d}$. It is easy to see that under this mapping strategy each column and each row processors form a subcube and the adjacent processors on the grid are directly connected.

The matrix elements are distributed among the processors by full column and row wrapmapping, i.e., matrix element $\boldsymbol{a}_{i j}$ is placed in processor row $\mathbf{I}$ and column $\mathbf{J}$ according to $\mathbf{I}=(i-1)$ $\bmod \mathrm{p},+\mathrm{l}$ and $\mathbf{J}=(\mathrm{j}-1) \bmod \boldsymbol{p}_{c}+1$. Thus $1 \leq \mathbf{I} \leq \boldsymbol{p}_{r}$ and $1 \leq \mathrm{J} \leq \boldsymbol{p}_{\boldsymbol{c}}$. This mapping strategy improves the computation load balance when interchanges are performed [1]. In our implementation, the objective is to achieve the overlap of communication and computation. This full column and row wrap-mapping strategy will balance both the computation and communication loads.

Under the submatrix partitioning strategy, there are two types of communication required at every iteration: one to pass the pivot row and another to pass the multipliers. The multipliers are the matrix column that is to be set to zero in that step. These data are available in one column of processors and the multipliers must be passed to the remaining processor columns. Therefore the communicirtion path for passing multipliers is across processor columns.

The pivot row is contained initially in a single row of processors. The communication path for passing pivot row is across processors rows. Before sent out, each element of the pivot row need to be normalized by the pivot element. The pivot element is contained in the multipliers passed to the processor. Therefore the normalization and the broadcast of the pivot row can not start until the processors get the multipliers.

We still use the compute-and-send-ahead strategy to achive the overlap of communication and computation, i.e., at the $\mathrm{k}$-th iteration, the data elements of the $\mathrm{k}+1$ st pivot row and multipliers are first updated and then sent out. Unlike in Algorithm I, where there is only one broadcast within 
the hypercube at every iteration, in Algorithm II, there are concurrent broadcasts within subcubes at each iteration: first there are $p$, concurrent broadcasts of segments of multipliers within the subcube rows; then there are $\boldsymbol{p}_{\boldsymbol{c}}$ concurrent broadcasts of segments of the pivot row within the subcube columns. For simplicity, we assume $p,=p_{c}=\sqrt{p}$. We use the notation $[\mathrm{k}]$ to represent $(\mathrm{k}-1) \bmod p_{\tau}+1$. Then at the kth iteration, processors in the $[\mathrm{k}+\mathrm{I}]-\mathrm{st}$ column and the $[\mathrm{k}+\mathrm{I}]-\mathrm{st}$ row are roots of the broadcast trees. As an example, Figure 7 shows the data flow for Algorithm II where $\boldsymbol{p}=16$ and $[\mathrm{k}+1]=2$.

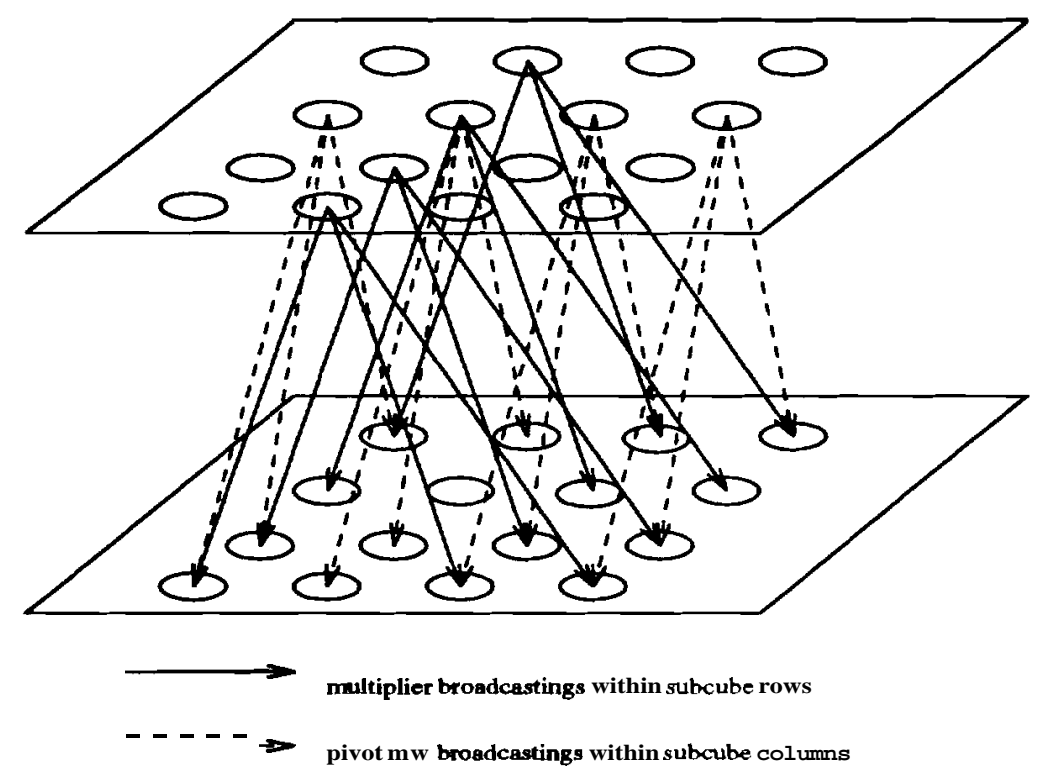

Figure 7: Data flow of Algorithm II.

Let $(\mathrm{I}, J)$ be the coordinates for processor $\mathrm{P}$ in the processor array, where $1 \leq \mathrm{I} \leq p_{r}$ and $\mathbf{1} \leq \mathrm{J} \leq p_{\mathrm{c}}$. The pseudo code for the kth step of the Algorithm II is as following:

if $(I \neq[k+1]$ and $\mathbf{J} \neq[k+1]$ then

update all the elements it has using the corresponding segments of the kth pivot row and multipliers;

Interrupt handling:

If the message is from a processor in the same processor row, pass it to its children nodes in the row subcube, if there is any; If the message is from a processor in the same processor column, pass it to its children nodes in the column subcube, if there is any :

if $(\mathrm{I}=[\mathrm{k}+1]$ and $\mathbf{J}=[\mathrm{k}+1]$ then updiste the corresponding segment of the $k+1$ st column of $A$ broadcast this column segment in the row subcube; 
update the corresponding segment of the $k+1$ st row of $A$;

and normalize it using the $k+1$ st pivot element;

brcadcast this row segment and the pivot elelment in the column subcube;

update the rest of the elements of $\mathrm{A}$;

if $(I \#[k+1]$ and $\mathbf{J}=[k+1]$ then

update the corresponding segment of the $k+1$ st column of $\mathrm{A}$;

broadcast this column segment in the row subcube;

upclate the rest of the elements of A.

Interrupt handling:

If the message is from a processor in the same processor row,

pass it to its children nodes in the row subcube, if there is any;

if $(I=[k+1]$ and $\mathbf{J} \neq[k+1]$ then

update the corresponding segment of the $k+1$ st row of $\mathrm{A}$;

update the rest of the elements of A;

Interrupt handling:

If the message is from a processor in the same processor column

pass it to its children nodes in the column subcube, if there is any;

normalize the corresponding segment of the $k+1$ st row $\mathrm{A}$

using the $k+1$ st pivot element;

broadcast this row segment and the pivot element in the row subcube;

In the above algorithm, whenever a message arrives at the processor, if the processor is doing computation, it will be interrupted and start executing the communication interrupt handling. If on the other hand, the processor has finished all the computation at a particular step and the message has not yet arrived, it will become idle until the message arrives, and then start executing the interrupt handling code before it goes to the next step of the loop.

The algorithm for each broadcast within a subcube-row or subcube-column is essentially the same as the broadcasting algorithm proposed in Section 4. Consider the i-th processor row consisting processors with physical addresses $\boldsymbol{g}_{i}^{d} \boldsymbol{g}_{1}^{d}, \boldsymbol{g}_{i}^{d} \boldsymbol{g}_{2}^{d}, \ldots, \boldsymbol{g}_{i}^{\boldsymbol{d}} \boldsymbol{g}_{2^{d}}^{d}$. From Section 4 we know now to construct ar family of $\mathrm{SBT}^{7} \mathrm{~s}$ :

$$
S B T(\operatorname{Gray}(d))=\left(S B T^{(1)}, S B T^{(2)}, \cdots, S B T^{\left(2^{d}\right)}\right),
$$

where in $\boldsymbol{S} \boldsymbol{B} \boldsymbol{T}^{(\boldsymbol{k})}, \boldsymbol{g}_{k}^{\boldsymbol{d}}$ is the root node and $\boldsymbol{g}_{k+1}^{d}$ is a leaf node at the first level.

A family of $\mathrm{SBT}^{7} \mathrm{~s}$ in the i-th processor row can be obtained by making the following transformation on each node of each SBT in $S B T(G r a y(d))$ : if the physical address of a node is $g_{j}$, then replace it with $g_{i}^{d} g_{j}, 1 \leq \mathbf{j} \leq 2^{d}$. Similarly a family of $\mathrm{SBT}^{7} \mathrm{~s}$ in the $\mathrm{j}$-th processor column can 
be obtained by the following transformation on each node of each SBT in $S B T(G r a y(d))$ : if the physical address of a node is $g_{i}$, then replace it with $g_{i} g_{j}^{d}, 1 \leq i \leq 2^{d}$. 


\section{Analysis of Algorithm II}

In this section we give the performance analysis of Algorithm II. For simpliciliy we only consider the case the hypercube dimension $\mathrm{d}$ is even, i.e., $p_{\mathrm{c}}=p,=\sqrt{p}=2^{\frac{\mathrm{d}}{2}}$. Let $P_{i j}$ be the processor in the ith processor row and $\mathrm{j}$ th processor column, $1 \leq \mathrm{i} \leq \sqrt{p}, 1 \leq \mathrm{j} \leq \sqrt{p}$. The following notations are used in the analysis:

$T_{\text {start }}^{k}\left(P_{i, j}\right): \quad$ The time $P_{i j}$ starts the Eth loop.

$T_{\text {complete }}^{k}\left(P_{i j}\right):$ The time $P_{i j}$ completes the kth loop.

$T^{k}\left(P_{i j}\right): \quad$ The computation load of $P_{i j}$ at the kth loop.

$T^{\prime}$ : $\quad$ The time to update or normalize one segment of the multipliers or the pivot row elements.

$T_{\text {send }-r}^{k}\left(P_{[k] j}\right):$ The time that the segment of kth row of A is sent by $P_{[k] j}$.

$t_{r}^{k}\left(P_{i j}\right): \quad$ The time it takes for this segment of the kth row to reach $P_{i j}$.

$T_{\text {send-c }}^{k}\left(P_{i[k]}\right)$ : The time that the segment of the kth column of $A$ is sent by $P_{i[k]}$.

$t_{c}^{k}\left(P_{i j}\right): \quad$ The time it takes for this segment of the $k$ th column to reach $P_{i j}$.

$\boldsymbol{s}_{r}^{\boldsymbol{k}}\left(P_{i j}\right): \quad$ The setup overhead of $P_{i j}$ when broadcasting the $\boldsymbol{k}$ th row segment.

$s_{c}^{k}\left(P_{i j}\right): \quad$ The setup overhead of $P_{i j}$ when broadcasting the kth column segment.

The following theorem shows that the total setup overhead of Algorithm II is twice that of Algorithm I.

Theorem 7 In Algorithm 11, the total setup overhead of each processor is $\mathrm{Nt}$,.

Proof: In Algorithm II, each processor involves $N$ one-to-all broadcasts in its subcube column and $N$ one-to-all broadcasts in its subcube row. By the construction of the SBT's, for consecutive $\sqrt{p}$ broadcastings, each processor incurs setup overhead of $\frac{1}{2} \sqrt{p} t_{s}$. Therefore the total setup overhead for each processor is $2 \cdot \frac{1}{2} \sqrt{p} t_{s} \frac{N}{\sqrt{p}}=N t_{s}$.

Each processor involves two broadcastings at every iteration. The situation, might occur that when a message containing the segment of the pivot row arrives at an intermediate processor, this processor is in the process of setting up the communication channel for a message containing the segment of the multipliers. Therefore the message can not he immediately pass over. Instead, it will be delayed until the processor finishes setting up the channel for the previous message. This situation can not happen in Algorithm I, as proved in Theorem 4. In the worst case, a message is delayed for a time of $t$, at an intermediate processor. As a result,, we have

$$
H\left(P_{[k] j}, P_{i j}\right)\left(t_{s}+t_{w} \frac{N}{\sqrt{p}}\right) \leq t_{r}^{k}\left(P_{i j}\right), t_{c}^{k}\left(P_{i j}\right) \leq H\left(P_{[k] j}, P_{i j}\right)\left(2 t_{s}+t_{w} \frac{N}{\sqrt{p}}\right)
$$


Lemma 5 We have ihe following recurrent relaiionship:

$$
\begin{aligned}
& T_{s t a r t}^{k}\left(P_{i j}\right)= \begin{cases}\max \left\{T_{c o m p l e t e}^{k-1}\left(P_{i j}\right)+s_{c}^{k}\left(P_{i j}\right)+s_{r}^{k}\left(P_{i j}\right), T_{s e n d-c}^{k}\left(P_{i[k]}\right)\right. & \\
\left.+t_{c}^{k}\left(P_{i j}\right)+s_{c}^{k}\left(P_{i j}\right), T_{s e n d-r}^{k}\left(P_{[k] j}\right)+t_{r}^{k}\left(P_{i j}\right)+s_{r}^{k}\left(P_{i j}\right)\right\}, & i \neq[k], j \neq[k], \\
\max \left\{T_{c o m p l e t e}^{k-1}\left(P_{[k] j}\right)+s_{c}^{k}\left(P_{[k] j}\right)+s_{r}^{k}\left(P_{[k] j}\right), T_{s e n d-c}^{k}\left(P_{[k][k]}\right)\right. & \\
\left.+t_{c}^{k}\left(P_{[k] j}\right)+s_{c}^{k}\left(P_{[k] j}\right)\right\}, & i=[k], j \neq[k], \\
\max \left\{T_{c o m p l e t e}^{k-1}\left(P_{i[k]}\right)+s_{c}^{k}\left(P_{i[k]}\right)+s_{r}^{k}\left(P_{i[k]}\right), T_{s e n d-r}^{k}\left(P_{[k][k]}\right)\right. & \\
\left.+t_{r}^{k}\left(P_{i[k]}\right)+s_{r}^{k}\left(P_{i[k]}\right)\right\}, & i \#[k], j=[k], \\
T_{\text {complete }}^{k-1}\left(P_{[k][k]}\right)+s_{c}^{k}\left(P_{[k][k]}\right)+s_{r}^{k}\left(P_{[k][k]}\right), & i=[k], j=[k] .\end{cases} \\
& T_{\text {complete }}^{k}\left(P_{i j}\right)=T_{\text {start }}^{k}\left(P_{i j}\right)+T^{k}\left(P_{i j}\right) \\
& T_{\text {send-c }}^{k}\left(P_{i[k]}\right)=T_{s t a r t}^{k-1}\left(P_{i[k]}\right)+T^{\prime} \\
& T_{s i n d-r}^{k}\left(P_{[k] j}\right)=\max \left\{T_{s t a r t}^{k-1}\left(P_{[k] j}\right)+T^{\prime}, T_{s e n d-c}^{k}\left(P_{[k][k]}\right)+t_{c}^{k}\left(P_{[k] j}\right)+s_{c}^{k}\left(P_{[k] j}\right)\right\}+T^{\prime}
\end{aligned}
$$

We say that communication is fully overlapped by computation if

$$
T_{\text {start }}^{k}\left(P_{i j}\right)=T_{\text {complete }}^{k-1}\left(P_{i j}\right)+s_{c}^{k}\left(P_{i j}\right)+s_{r}^{k}\left(P_{i j}\right), \quad 2 \leq k \leq N, \quad \forall i, j
$$

Theorem 8 When $\mathrm{N} \geq N_{0}$, ihe communicaiion can be fully overlapped by computation, where $N_{0}$ is ihe positive rooi of ihe following quadraiic equaiion:

$$
\left(\frac{N^{2}}{p}-\frac{3 N}{\sqrt{p}}\right) f=2 \sqrt{p} t_{s}+2\left(2 t_{s}+t_{w} \frac{N}{\sqrt{p}}\right) \log p .
$$

Proof: See Appendix.

At the beginning of Algorithm II, each processor except $P_{11}$ must be initially idle until it got the multipliers and/or the first pivot row elements. Let $d_{0}\left(P_{i j}\right)$ be the initial delay of $P_{i j}$.

Corollary 4 When $\mathrm{N} \geq N_{0}$, ihe communicaiion overhead of processor $P_{i j}$ :in Algorithm II is $d_{0}\left(P_{i j}\right)+N t_{s}$.

Next we give the simulation results on the parallel overhead of Algorithm II, obtained by numerically evaluating the recurrent relationships give in Lemma 5 . The parameters ( $\mathrm{f}$, $\mathrm{t}$, and $\mathrm{t}$ ) are the same as in Section 5. Figure 8 depicts the communication overhead $T_{\text {comm }}$ vs the matrix size 
$\mathrm{N}$ when the machine size $\mathrm{p}=16$. As a comparison, we put the corresponding curve of Algorithm I (curve I. in Figure 5) in the same figure. We can see that when the matrix size $\mathrm{N}$ is small, Algorithm II incurs less overhead than Algorithm I; but when $\mathrm{N}$ becomes large, Algorithm I incurs much less overhead than Algorithm II. We can also see that in Algorithm II the: matrix size $N_{0}$ for full overlap is smaller than that in Algorithm I. Figure 9 shows the communication overheads with different machine sizes. We can see that when full overlap is achieved, the communication overhead is independent of the machine size (if we neglect the initial delay), and linearly increases with the problem size (i.e., $T_{\text {comm }}=\mathrm{Nt}$,).

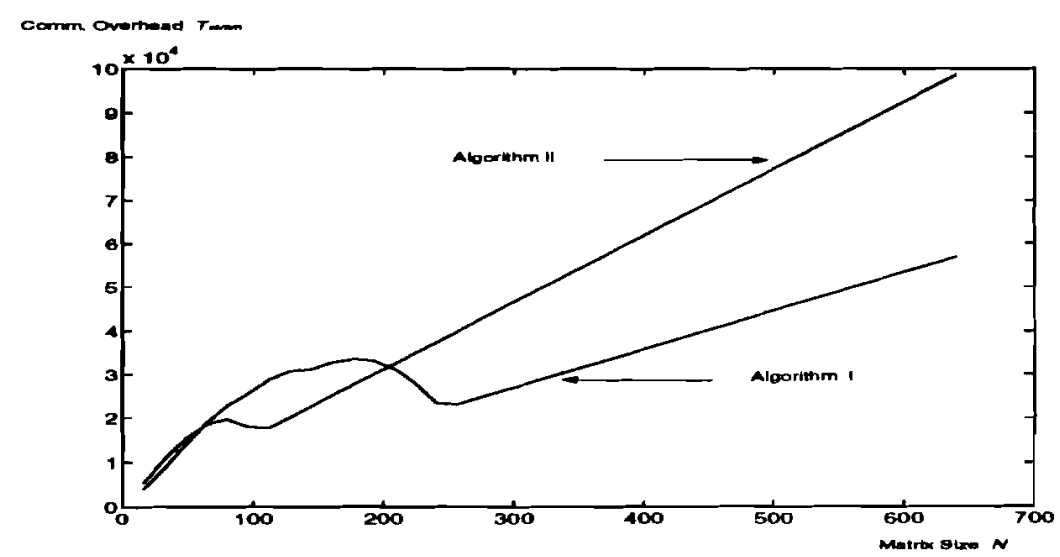

Figure 8: Communication overheads of Algorithm II (Number of processor $\mathrm{p}=16$ ).

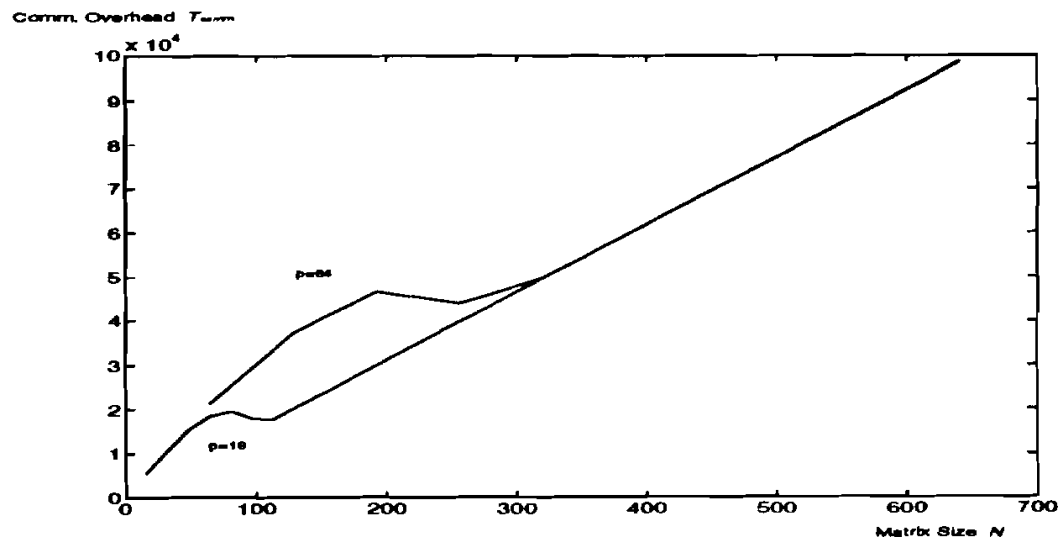

Figure 9: Communication overheads of Algorithm II with different machine sizes. 


\section{Algorithm III: Submatrix Partitioning, With Pivoting}

We consider the parallel GJ inversion algorithm with column interchanges. Since the matrix is partitioned by submatrix, the searching of pivot element can not be done by a single processor. Instead, a row of processors each determine the local pivot element and then find the pivot element through recursive doubling. During the E-th iteration, the $(E+1)$ st pivot row elements can be computed ahead and sent ahead by a row of processors; but the multipliers can not be computed before the processor gets the segment of the pivot row and finds out the local pivot element. In Algorithm. III, we let each processor updates its share of the submatrix colurnn by column. As soon as the message containing the pivot row elements arrives, it will be interrupted and find the local pivot element. Then it will first update the column segment corresponding to this local pivot element and then participate permutation among the processors in the same subcube-row. After $\frac{d}{2}$ write-read pairs, each processor will get the pivot element and the corresponding segments of multiplier:; Then it will nomarlize the pivot row elements using the pivot element.

Therefore at each iteration of Algorithm III, each processor will be interrupted $\frac{d}{2}+1$ times: one for the broadcasting of the pivot row elements; $\frac{d}{2}$ for the permutations. We have the following theorem regarding the total setup overhead of Algorithm III.

Theorem 9 In Algorithm 111, the setup overhead for each processor is $\frac{1}{2}(1+\log p) N t_{s}$.

Proof: The one-to-all broadcastings of the pivot row elements within the. subcube-columns causes setup overhead of $\frac{1}{2} N t_{s}$. At each iteration, the permutation within the subcube-rows to find the pivot elements causes each processor a setup overhead of $\frac{1}{2} \log p t_{s}$, and there are totally $\mathrm{N}$ iterations. So the overall setup overhead for each processor is $\frac{1}{2}(1+\log \mathrm{p}) \mathrm{Nt}$, .

Therefore Algorithm III incurs much larger setup overhead than Algorithm I and Algorithm II. Further more, Since the recursive doubling essentially causes synchronization barrier for all the processors, although we can use the interrupt handling capability to let the processor do some computation while waiting for the message, it is difficult to achieve full overlap.

if $(\mathrm{I}=[\mathrm{E}+1])$ then

update the corresponding segment of the $(E+1)$ st row of A

broadcast this row segment in the subcube-column;

update the rest of the submatrix of A column by column;

if $(I \neq[E+1])$ then

update the share of the submatrix of A column by column;

Interrupt handling:

if the message is from a processor in the subcube column, then

forward this message if needed;

find the local pivot element;

update the segment column corresponding to the local pivot; 
write this updated segment to its first neighbor in the subcub e-raw;

if the message is from the $\mathrm{jth}$ neighbor in the subcube-row,then

if $\mathbf{j}<\frac{d}{2}$ then find the larger pivot element and send the corresponding segment to its $\mathbf{j}+1$-th neighbor;

if $\mathbf{j}=\frac{d}{2}$ then find the larger pivot element and the corresponding segment is the multiplier. 


\section{Scalability Analysis}

The scalability of a parallel algorithm on a parallel architecture is a measure of its capability to effectively utilize the processors. Several metrics have been proposed in the literature to measure the scalability of algorithms and machine systems $[28,29,30]$ In this section we adopt two metrics, namely, the isoefficiency metric [28] and the [30] metric to study the scalability of the parallel Gauss - Jordan matrix inversion algorithm proposed in this report.

\subsection{The Isoefficiency Metric of Scalability}

Let $\mathrm{p}$ be the number of processors, $\mathrm{W}$ be the problem size which is the total amount of computation to be done, and $T_{o}$ be the total parallel overhead of all the processors. Then the execution time on $p$ processors $T_{p}$ satisfies $\mathrm{W}+T_{o}=p T_{p}$. Speedup is defined as $\mathrm{S}=\frac{W}{T_{p}}$. Efficiency is defined as $E=\frac{S}{\mathrm{P}}=\frac{W}{p T_{p}}=\frac{W}{W+T_{o}}=\frac{1}{1+\frac{T_{o}}{W}}$.

Gupta and Kumar defined an isoefficiency function $f_{E}(p)$ to measure the scalability of parallel algorithm and architecture combinations $[28,29]$, where $f_{E}(p)$ is the amount of work needed to maintain an constant efficiency $\boldsymbol{E}$ when p processors are used.

Theorem.10 For the parallel GJ inversion algoriihm on a hypercube architecture, when the mairix is partitioned as submairices and ihere is no overlap beiween cornmunicaiion and compuialion (i.e., Algoriihm 0), ihen the isoefficiency function $f_{E}(p)=\Theta\left(p^{\frac{3}{2}}(\log p)^{3}\right)$.

Proof: When submatrix partitioning is used and if there is no overlapping between communication and computation, the communication overhead for each processor is $N\left(t_{s}+t_{w} \frac{N}{\sqrt{p}}\right) \log$. Therefore the total communication overhead is $T_{o}=p N\left(t_{s}+t_{w} \frac{N}{\sqrt{p}}\right) \operatorname{logp}$. The work load of the GJ inversion algorithm is $\mathrm{W}=N^{3}$ f. To keep the efficiency $\boldsymbol{E}$ fixed, we have $f_{E}(p)=\mathrm{W}=K T_{o}$, where $K=\frac{E}{1-E}$. Therefore $N^{3} \mathrm{f}=K p N\left(t_{s}+t_{w} \frac{N}{\sqrt{p}}\right) \operatorname{logp}$, or $\mathrm{f} \mathrm{N}^{2}-K t_{w} \sqrt{p} \log p N-K t_{s} p \log \mathrm{p}=0$ Solving for: $\mathrm{N}$ we get $\mathrm{N}=\Theta(\sqrt{p} \log p)$. Thus $f_{E}(p)=\mathrm{W}=\mathrm{N}^{3} \mathrm{f}=\Theta\left(p^{\frac{3}{2}}(\log p)^{3}\right)$.

Theorem 11 For the parallel GJ inversion algoriihm on a hypercube architecture, when the mairix is partitioned by rows and when communicaiion is fully overlapped by computation (i.e., Algorithm I), ihen ihe isoefficiency funclion $f_{E}(p)=\Theta\left(p^{\frac{3}{2}}\right)$.

Proof: When the matrix is partitioned by rows and when communication is fully overlapped by computation, the overhead for each processor is $\frac{1}{2} N t_{s}$. Therefore the total overhead is $T_{o}=\frac{1}{2} p N t_{s}$. To keep the efficiency $\boldsymbol{E}$ fixed, we have $f_{E}(p)=\mathrm{W}=K T_{0}$, where $K=\frac{E}{1-E}$. Therefore $\mathrm{N}^{3} \mathrm{f}=$ $\frac{1}{2} K p N t_{s}$. Solving for $\mathrm{N}$ we get $\mathrm{N}=\sqrt{\frac{K t_{s}}{2 f}}=\Theta(\sqrt{p})$. Thus $f_{E}(p)=\mathrm{W}=N^{3} \mathrm{f}=\Theta\left(p^{\frac{3}{2}}\right)$.

\subsection{The Isospeed Metric of Scalability}

In [30] the scalabilty of a parallel algorithm-machine combination is defined based on the isospeed metric. The average speed is the achieved speed of the given computing system divided by $p$, 
the number of processors. Let $W$ be the amount of work of an algorithm when $p$ processors are employed, and $W^{\prime}$ be the amount of work of the algorithm when $\boldsymbol{p}^{\prime}>p$ processors are employed to maintain the average speed, then the scalabiliiy from sysiem size $p$ to system size $p^{\prime}$ of the algorithm - machine combination is defined as: $\phi\left(p, p^{\prime}\right)=\frac{\frac{W^{p}}{p}}{\frac{W^{\prime}}{p^{\prime}}}$. The work $W^{\prime}$ is determined by the isospeed constraint.

Theorem 12 For Algorithm I, when ihe problem size is such that communication is fully overlapped by computation, then the scalabiliiy from sysiem size $p$ to system size $p^{\prime}\left(p^{\prime}>p\right)$ is $\phi\left(p, p^{\prime}\right)=\sqrt{p^{\prime}}$.

Proof: $\boldsymbol{B} \boldsymbol{y}$ the isospeed constraint, we have $\frac{W}{p T_{p}}=\frac{W^{\prime}}{p^{\prime} T_{p^{\prime}}}$, or $\frac{W}{W+T_{o}}=\frac{W^{\prime}}{W^{\prime}+T_{o}^{\prime}}$, or $\frac{1}{1+\frac{T_{o}}{W}}=\frac{1}{1+\frac{T_{o}^{\prime}}{W^{\prime}}}$.

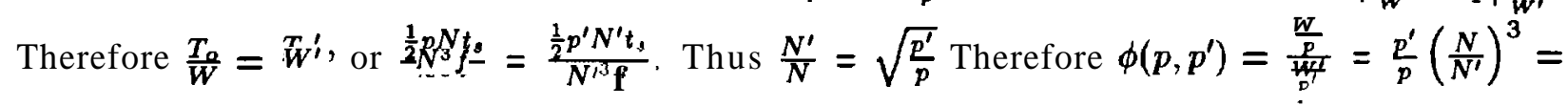
$\frac{p^{\prime}}{p}\left(\frac{p}{p^{\prime}}\right)^{\frac{3}{2}}=\sqrt{\frac{p}{p}}$ 


\section{LIJ Factorization}

In this section, we apply the ideas of overlapping communication and computation, to the parallel LU factorization algorithm, using the row partitioning strategy and column interchanges for partial pivoting.

The pseudocode for the sequential row oriented LU decomposition algorithm is given below:

$$
\begin{aligned}
& \text { for } k=1 \text { to } N-1 \\
& \text { for } i=k+1 \text { to } N \\
& \qquad \begin{array}{r}
l_{i k}=a_{i k} / a_{k k} \\
\text { for } j=k+1 \text { to } N \\
a_{i j}=a_{i j}-l_{i k} a_{k j}
\end{array}
\end{aligned}
$$

The $\mathbf{j}$ loop subtracts multipliers of the $\boldsymbol{k}$ th row of the current A from succeeding rows. At the kth stage of the algorithm, there are $(\mathrm{N}-\mathrm{k})$ row updatings, and the length of each row to be updated is $(\mathrm{N}-\mathrm{k}$ ). (Recall in the GJ inversion algorithm, at the $\boldsymbol{k}$ th stage, there are $\mathrm{N}$ row updatings., and each row is of length N.)

When implemented in parallel, to keep the computational load balanced among the processors, we use a variation of the row wrapped storage - reflection wrapped storage. Here the rows are distributed as illustrated in Figure 10 for the case of $p=4$ and $N=16$. In general, the first $p$ rows are distributed to the p processors in order, the next $\mathrm{p}$ rows are distributed in reverse order, and so on. We use the notation $P_{\{k\}}$ to denote the processor that has the kth row.
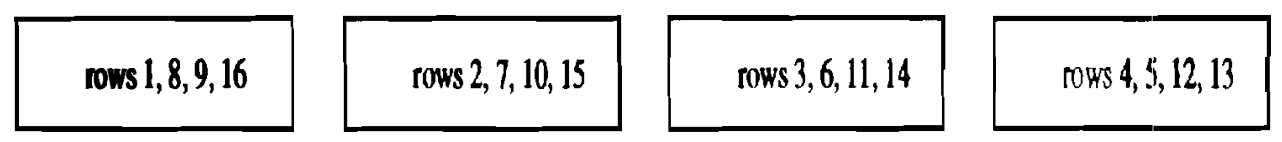

Figure 10: Reflection wrapped row storage.

The parallel LU factorization algorithm using row partitioning is similar to the Algorithm I in Section 3. During the k-th iteration, each processor first gets the k-th pivot row of A and the pivot element from processor $P_{\{k\}}$, and then does the row updatings. To overlap communication and computation, processor $P_{\{k+1\}}$ first updates the $(\mathrm{k}+1)$-st row of A during the k-th iteration. Then it finds the pivot element and normalizes this row (compute-ahead). After that it broadcasts this $(\mathrm{k}+1)$-st pivot row and the pivot element to all the other processors, before it resumes to update the other rows using the k-th pivot row (send-ahead).

Consider the computation load of each processor at the $k$ th stage. Let $q^{\prime}=\left\lfloor\frac{k}{p}\right\rfloor$, then the computation load of processor $\mathbf{P}$ at the kth stage is

$$
T^{k}(P)= \begin{cases}(n-q-1)(N-k) f, & P \in\left\{P_{\{p q+1\}}, P_{\{p q+2\}}, \cdots, P_{\{k\}}\right\} \\ (n-q+1)(N-k) f, & P=P_{\{k+1\}}, \\ (n-q)(N-k) f, & P \in\left\{P_{\{k+2\}}, \cdots, P_{\{p(q+1)\}}\right\}\end{cases}
$$


Lemma 1 For any $1 \leq K \leq N$, and for any two processors $P$ and $P^{\prime}$

$$
\left|\sum_{k=1}^{K} T^{k}(P)-\sum_{k=1}^{K} T^{k}\left(P^{\prime}\right)\right|<3 p N f
$$

Proof: Without loss generality we assume $P=P_{\{i\}}$ and $P^{\prime}=P_{\{j\}}$, where $1 \leq \mathrm{i}<j \leq p$. Consider $\sum_{k=1}^{2 p} T^{k}(P)$.

If $i \neq 1$ and $i \neq p$, then

$$
\begin{aligned}
\sum_{k=1}^{p} T^{k}(P) & =\sum_{k=1}^{i-2} T^{k}(P)+T^{i-1}(P)+\sum_{k=i}^{p} T^{k}(P) \\
& =\sum_{k=1}^{i-2} n(N-k) f+(n+1)[N-(i-1)] f+\sum_{k=i}^{p}(n-1)(N-k) f \\
& =\sum_{k=1}^{p}(n-1)(N-k) f+[N-(i-1)] f+\sum_{k=1}^{i-1}(N-k) f . \\
\sum_{k=p+1}^{2 p} T^{-k}(P) & =\sum_{k=p+1}^{p+(p-i)-1} T^{k}(P)+T^{p+(p-i)}(P)+\sum_{k=p+(p-i)+1}^{2 p} T^{k}(P) \\
& =\sum_{k=p+1}^{p+i)-1}(n-1)(N-k) f+n[N-(2 p-i)] f+\sum_{k=p+(p-i)+1}^{2 p}(n-2)(N-k) \\
& =\sum_{k=p+1}^{2 p}(n-2)(N-k) f+[N-(2 p-i)] f+\sum_{k=p+1}^{p+(p-i)}(N-k) f .
\end{aligned}
$$

Thus

$$
\begin{aligned}
\sum_{i=1}^{2 p} T^{k}(P)= & \sum_{k=1}^{p}(n-1)(N-k) f+\sum_{k=p+1}^{2 p}(n-2)(N-k) f+(2 N+1-2 p) f \\
& +\sum_{k=1}^{i-1}(N-k) f+\sum_{k=p+1}^{p+(p-i)}(N-k) f .
\end{aligned}
$$

If $\mathbf{i}=\geqq$ then

$$
\begin{aligned}
\sum_{k=1}^{2 p} T^{k}(P)= & \sum_{k=1}^{p}(n-1)(N-k) f+\sum_{k=p+1}^{2 p-2}(n-1)(N-k) f+n[N-(2 p-1)] f+(n-1)[N-(2 p)] f \\
= & \sum_{k=1}^{p}(n-1)(N-k) f+\sum_{k=p+1}^{2 p}(n-2)(N-k) f+(2 N+1-4 p) f \\
& +\sum_{k=p+1}^{p+(p-i)}(N-k) f .
\end{aligned}
$$


Therelore we have

$$
\begin{aligned}
& \left|\sum_{k=1}^{2 p} T^{k}(P)-\sum_{k=1}^{2 p} T^{k}\left(P^{\prime}\right)\right| \\
= & \begin{cases}|-2 p(j-i) f| & \text { if } i \neq 1, \\
|-2 p(j-i+1) f| & \text { if } i=1 .\end{cases} \\
\leq & 2 p^{2} f .
\end{aligned}
$$

Similarly we can show that for any $r \geq 0$,

$$
\left|\sum_{k=2 p r}^{2 p(r+1)} T^{k}(P)-\sum_{k=2 p r}^{2 p(r+1)} T^{k}\left(P^{\prime}\right)\right| \leq 2 p^{2} f .
$$

Therefore

$$
\begin{aligned}
& \left|\sum_{k=1}^{K} T^{k}(P)-\sum_{k=1}^{K} T^{k}\left(P^{\prime}\right)\right| \\
\leq & 2 p^{2} f\left\lfloor\frac{K}{2 p}\right\rfloor+\left|\sum_{k=\left\lfloor\frac{K}{2 p}\right\rfloor 2 p+1}^{K}\left[T^{k}(P)-T^{k}\left(P^{\prime}\right)\right]\right| \\
< & 2 p^{2} \frac{K}{2 p}+2 p N f \\
\leq & 3 N f .
\end{aligned}
$$

Unlike the parallel GJ inversion algorithm, in the parallel LU decomposition algorithm, the computation load of each processor decreases as the computation proceeds. When the computation load decreases to a certain level, there will not be enough computation to do to compensate for the processor :idle time caused by data communication. Therefore full overlap of communication and computation can not be achieved throughout the whole computation process. Nevertheless, it can be shown that for a certain number $\mathrm{c}$, where $0<\mathrm{c}<1$, in the parallel LU factorization algorithm, up to stage $c N$, full overlap of communication and computation can be achieved, given the matrix size $\mathrm{N}$ is large enough, as stated by the following theorem.

Theorem 13 When $N>N_{0}$, up to the $c N$ th iteration, where $0<c<1$, full overlap of communication and computation can be achieved, where $N_{0}$ is the positive root of the following quadratic equation.

$$
\left[\frac{N\left(1-c^{2}\right)}{p}-3 p\right] N f=\frac{1}{2} p t_{s}+2\left(t_{s}+t_{w} N\right) \log p
$$

Proof: Using the above lemma, the proof is similar to the proof of Theorem 5 and therefore omitted here. 
Next we give the simulation results on the processor idle time vs the number of iterations for the parallel LU decomposition algorithm. The machine parameters are the same as in the previous sections. In Figure 11 the two curves correspond to $\mathrm{N}=160$ and $\mathrm{N}=320$ respectively, and $\mathrm{p}=8$. We can see for $\mathrm{N}=160$, up to $\mathrm{k}=55$, full overlap is achieved; while for $\mathrm{N}=320$, up to $k=215$, full overlap is achieved.

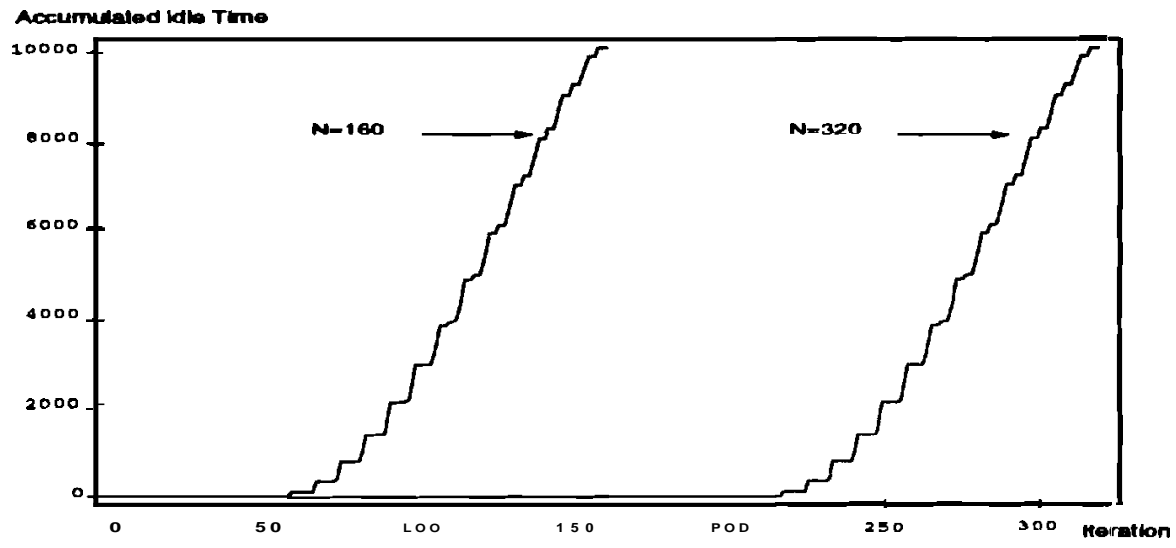

Figure 11: The accumulated processor idle time vs. the number of iterations in the parallel LU decomposition algor:thm $(p=8)$. 


\section{Concluding Remarks}

Most parallel matrix algorithms proposed in the literature do not attempt to overlap inter-processor communication by computation. This leads to increased communication overhead, and might lead to conclusions that are not valid when communication overhead is systematically minimized. For example, it is claimed in $[1,3]$ that the submatrix partitioning strategy for GJ algorithm is superior to the row/column partitioning strategy because of lower communication overhead. We, however, show that if communication and computation are efficiently overlapped, then the row partitioning strategy has a lower communication overhead than the submatrix partitioning strategy.

We first proposed a new broadcasting algorithm on the hypercube multiprocessor for parallel GJ algorithm. This algorithm ensures that the data are sent out from the source and arrives at the destinations at the earliest possible time. We then gave the parallel GJ inversion algorithm using row partitioning. We prove a lower bound on the matrix size such that data transmission is fully overlapped by computation. We also prove that the message length in the input buffer of each processor is at most 2 .

We also consider the algorithms under submatrix partitioning, with or without pivoting. We show that when submatrix partitioning is used, even when the communication is fully overlapped by compuration, the communication overhead is larger than when using row partitioning.

Our numerical simulation shows that when the algorithms proposed in this report is used, the parallel algorithms incurs much less communication overhead compared with the algorithm in [1], even when the communication is not completely overlapped by computation. Finally, we observe that the icleas in this report can be applied to other parallel algorithms as well, such as parallel LU factorization algorithm. 


\section{Appendix: Proof of Theorem 8}

Proof: Let

$$
\begin{aligned}
& \Delta T_{c}^{k}\left(P_{i j}\right)=\left(T_{\text {complete }}^{k-1}\left(P_{i j}\right)+s_{c}^{k}\left(P_{i j}\right)+s_{\tau}^{k}\left(P_{i j}\right)\right)-\left(T_{s e n d-c}^{k}\left(P_{i[k]}\right)+t_{c}^{k}\left(P_{i j}\right)+s_{c}^{k}\left(P_{i j}\right)\right), \\
& \Delta T_{\tau}^{k}\left(P_{i j}\right)=\left(T_{\text {complete }}^{k-1}\left(P_{i j}\right)+s_{c}^{k}\left(P_{i j}\right)+s_{\tau}^{k}\left(P_{i j}\right)\right)-\left(T_{s e n d-r}^{k}\left(P_{[k] j}\right)+t_{\tau}^{k}\left(P_{i j}\right)+s_{\tau}^{k}\left(P_{i j}\right)\right),
\end{aligned}
$$

We will show $\Delta T_{c}^{k}\left(P_{i j}\right) \geq 0$ and $\Delta T_{r}^{k}\left(P_{i j}\right) \geq 0, \forall i, j, 2 \leq \mathrm{k} \leq \mathrm{N}$. The proof is by induction on $k$.

(1) Baise case: $\mathbf{k}=\mathbf{2}$.

First consider the case $i \neq 2$ and $j \neq 2$.

$$
\Delta T_{r}^{2}\left(P_{i j}\right)=\left(T_{\text {complete }}^{1}\left(P_{i j}\right)+s_{c}^{2}\left(P_{i j}\right)+s_{r}^{2}\left(P_{i j}\right)\right)-\left(T_{\text {send }-\tau}^{2}\left(P_{2 j}\right)+t_{r}^{2}\left(P_{i j}\right)+s_{r}^{2}\left(P_{i j}\right)\right)
$$

If $T_{\text {send-r }}^{2}\left(P_{2 j}\right)=T_{s e n d-c}^{2}\left(P_{22}\right)+t_{c}^{2}\left(P_{2 j}\right)+s_{c}^{2}\left(P_{2 j}\right)+T^{\prime}$, then

$$
\begin{aligned}
& \Delta T_{r}^{2}\left(P_{i j}\right) \\
= & \left(T_{s t a r t}^{1}\left(P_{i j}\right)+T^{1}\left(P_{i j}\right)+s_{c}^{2}\left(P_{i j}\right)\right)-\left(T_{s e n d-c}^{2}\left(P_{22}\right)+t_{c}^{2}\left(P_{2 j}\right)+s_{c}^{2}\left(P_{2 j}\right)+T^{\prime}+t_{r}^{2}\left(P_{i j}\right)\right) \\
= & \left(T_{s t a r t}^{1}\left(P_{i j}\right)+T^{1}\left(P_{i j}\right)+s_{c}^{2}\left(P_{i j}\right)\right)-\left(T_{s t a r t}^{1}\left(P_{22}\right)+T^{\prime}+t_{c}^{2}\left(P_{2 j}\right)+s_{c}^{2}\left(P_{2 j}\right)+T^{\prime}+t_{r}^{2}\left(P_{i j}\right)\right) \\
= & \left(T^{1}\left(P_{i j}\right)-2 T^{\prime}\right)+s_{c}^{2}\left(P_{i j}\right)-\left(T_{s t a r t}^{1}\left(P_{22}\right)-T_{s t a r t}^{1}\left(P_{i j}\right)\right)-\left(t_{c}^{2}\left(P_{2 j}\right)+s_{c}^{2}\left(P_{2 j}\right)+t_{r}^{2}\left(P_{i j}\right)\right) \\
\geq & \left(\frac{N^{2}}{p}-\frac{2 N}{\sqrt{p}}\right) f-\left(\frac{N}{\sqrt{p}} f+\left(t_{s}+t_{w} \frac{N}{\sqrt{p}}\right) \log _{2} p\right)-\left(t_{s}+t_{w} \frac{N}{\sqrt{p}}\right) \log _{2} p \\
= & \left(\frac{N^{2}}{p}-\frac{3 N}{\sqrt{p}}\right) f-2\left(t_{s}+t_{w} \frac{N}{\sqrt{p}}\right) \log _{2} p \\
\geq & 0 .
\end{aligned}
$$

If $T_{s e n d-r}^{2}\left(P_{2 j}\right)=T_{s t a r t}^{1}\left(P_{2 j}\right)+2 T^{\nu}$, then

$$
\begin{aligned}
& \Delta T_{r}^{2}\left(P_{i j}\right) \\
= & \left(T_{s t a r t}^{1}\left(P_{i j}\right)+T^{1}\left(P_{i j}\right)+s_{c}^{2}\left(P_{i j}\right)\right)-\left(T_{s t a r t}^{1}\left(P_{2 j}\right)+2 T^{\prime}+t_{r}^{2}\left(P_{i j}\right)\right) \\
= & \left(T^{1}\left(P_{i j}\right)-2 T^{\prime}\right)+s_{c}^{2}\left(P_{i j}\right)-\left(T_{s t a r t}^{1}\left(P_{2 j}\right)-T_{s t a r t}^{1}\left(P_{i j}\right)\right)-t_{r}^{2}\left(p_{i j}\right) \\
\geq & \left(\frac{N^{2}}{p}-\frac{2 N}{\sqrt{p}}\right) f-\left(\frac{N}{\sqrt{p}} f+\left(t_{s}+t_{w} \frac{N}{\sqrt{p}}\right) \log _{2} p\right)-\frac{1}{2}\left(t_{s}+t_{w} \frac{N}{\sqrt{p}}\right) \log _{2} p \\
= & \left(\frac{N^{2}}{p}-\frac{3 N}{\sqrt{p}}\right) f-\frac{3}{2}\left(t_{s}+t_{w} \frac{N}{\sqrt{p}}\right) \log _{2} p \\
\geq & 0 .
\end{aligned}
$$

Similarly we can show $\Delta T_{r}^{2}\left(P_{i j}\right) \geq \mathbf{0}$ when $i=2$ or $\mathbf{j}=2$. And similarly we can show $\Delta T_{c}^{2}\left(P_{i j}\right) \geq 0, \forall i, j$.

(2) Induction Step: Assume the proposition is true for $2 \leq l \leq k$. We prove it is also true for $l=k+1$. We just show the case $i \neq \mathrm{k}+1$ and $j \neq \mathrm{k}+1$, and $T_{\text {send-r }}^{k+1}=T_{s e n d-c}^{k+1}\left(P_{[k+1][k+1]}\right)+$ $\left.t_{c}^{k+1}\left(P_{[k+1] j}\right)+s_{c}^{k+1}\left(P_{[k+1] j}\right)+T\right)$. The proofs for the other cases are similar. 
By the induction hypothesis, $T_{s t a r t}^{l}\left(P_{i j}\right)=T_{\text {complete }}^{l-1}\left(P_{i j}\right)+s_{c}^{k}\left(P_{i j}\right)+s_{r}^{k}\left(P_{i j}\right)$, for $2 \leq \mathrm{I} \leq \mathrm{k}$, Vi,j.

$$
\begin{aligned}
& \Delta T_{r}^{k+1}\left(P_{i j}\right) \\
= & \left(T_{\text {complete }}^{k k}\left(P_{i j}\right)+s_{c}^{k+1}\left(P_{i j}\right)+s_{r}^{k+1}\left(P_{i j}\right)\right)-\left(T_{s e n d-r}^{k+1}\left(P_{i j}\right)+s_{r}^{k+1}\left(P_{i j}\right)\right) \\
= & \left(T_{s t a r t}^{k}\left(P_{i j}\right)+T^{k}\left(P_{i j}\right)+s_{c}^{k+1}\left(P_{i j}\right)\right)-\left(T_{s e n d-c}^{k+1}\left(P_{[k+1][k+1]}\right)+t_{c}^{k+1}\left(P_{[k+1] j}\right)\right. \\
& \left.+s_{c}^{k+1}\left(P_{[k+1] j}\right)+T^{\prime}+t_{r}^{k+1}\left(P_{i j}\right)\right) \\
= & \left(r_{s t a r t}^{1}\left(P_{i j}\right)+\sum_{l=1}^{k} T^{l}\left(P_{i j}\right)+\sum_{l=1}^{k}\left(s_{c}^{l}\left(P_{i j}\right)+s_{r}^{l}\left(P_{i j}\right)\right)+s_{r}^{k+1}\left(P_{i j}\right)\right) \\
& -\left(T_{s t a r t}^{1}\left(P_{[k+1][k+1]}\right)+\sum_{l=1}^{k} T^{l}\left(P_{[k+1][k+1]}\right)-T^{k}\left(P_{[k+1][k+1]}\right)+T^{\prime}\right) \\
& -\left(\sum_{l=1}^{k}\left(s_{c}^{l}\left(P_{[k+1][k+1]}\right)+s_{r}^{l}\left(P_{[k+1][k+1]}\right)\right)+t_{c}^{k+1}\left(P_{[k+1] j}\right)+s_{c}^{k+1}\left(P_{[k+1] j}\right)+T^{\prime}+t_{r}^{k+1}\left(P_{i j}\right)\right) \\
= & T^{k}\left(P_{[k+1][k+1]}\right)+\sum_{l=1}^{k}\left(T^{l}\left(P_{i j}\right)-T^{l}\left(P_{[k+1][k+1]}\right)\right)-2 T^{\prime}-\left(T_{s t a r t}^{1}\left(P_{[k+1][k+1]}\right)-T_{s t a r t}^{1}\left(P_{i j}\right)\right) \\
& -\sum_{l=1}^{k}\left[\left(s_{c}^{l}\left(P_{[k+1][k+1]}\right)+s_{r}^{l}\left(P_{[k+1][k+1]}\right)-\left(s_{c}^{l}\left(P_{i j}\right)+s_{r}^{l}\left(P_{i j}\right)\right)\right]\right. \\
& -\left(t_{c}^{k+1}\left(P_{[k+1] j}\right)+s_{c}^{k+1}\left(P_{[k+1] j}\right)\right)-t_{r}^{k+1}\left(P_{i j}\right)+s_{r}^{k+1}\left(P_{i j}\right) \\
\geq & \left(\frac{N^{2}}{p}-\frac{2 N}{\sqrt{p}}\right) f-\left(\frac{N}{\sqrt{p}} f+\left(t_{s}+t_{w l} \frac{N}{\sqrt{p}}\right) \log _{2} p\right)-2 \sqrt{p} t_{s}-\left(t_{s}+t_{w} \frac{N}{\sqrt{p}}\right) \log _{2} p \\
\geq & \left(\frac{N^{2}}{p}-\frac{3 N}{\sqrt{p}}\right) f-2 \sqrt{p} t_{s}-2\left(t_{s}+t_{w} \frac{N}{\sqrt{p}}\right) \log _{2} p \\
\geq & 0 .
\end{aligned}
$$

Similarly we can show that $\Delta T_{c}^{k+1}\left(P_{i j}\right) \geq 0, \mathbf{V i}, \mathbf{j} \cdot \square$ 


\section{References}

[1] E. Chu, A. George and D. Quesnel, "Parallel matrix inversion on a subcube-grid ", Parallel Computing, 19, 1993, pp.243-256.

[2] T.J. Dekker and W.Hoffmann, "Rehabilitation of the Gauss-Jordan algorithm ", Numer. Math., 54, 1989, pp.591-599.

[3] M. Angelaccio and M. Colajanni, "Unifying and optimizing parallel linear algebra algorithms ", IEEE Transactions on Parrallel And Distributed Systems, Vol.4, No.12, Dec 1993, pp.13821397.

[4] J.M. Ortega, Introduction to Parallel and Vector Solution of Linear Systems, New York: Plenum, 1988.

[5] J.M. Ortega and C.H. Romine, "The ijk forms of factorization methods II. Parallel systems ", Parallel Computing, 7, 1988, pp.149-162.

[6] S.L. Johnsson, "Minimizing the communication time for matrix multiplication on multiprocessors", Parallel Computing, 19, 1993, pp.1235-1257.

[7] S.L. Johnsson and C-T Ho, "Optimum broadcasting and personalized cornmunication in hypercubes ", IEEE Transactions on Computers, Vol.38, No.9. Sept 1989, pp.1249-1268.

[8] E. Chu and A. George, "A balanced submatrix merging algorithm for multiprocessor architectures ", Parallel Computing, 18, 1992, pp.1-10.

[9] A.C. Elster and A.P. Reeves, "Block-matrix operations rising orthogonal trees ", Third Conf. on Hypercube Concurrent Computers and Applications, ACM, 1088, pp.1554-1561.

[10] A. Gerasoulis, I. Nelken, N. Missirlis and R. Peskin, "Implementing Gauss-Jordan on a hypercube multiprocessor", Third Conf. on Hypercube Concurrent Computers and Applications, ACM, 1988, pp.1569-1576.

[11] G.C. Fox, W. Furmanski and D.W. Walker, "Optimal matrix algorithms on homogeneous hypercubes ", Third Conf. on Hypercube Concurrent Computers and Applications, ACM, 1988, pp.1554-1561.

[12] P.G. Hipes and A. Kuppermann, "Gauss-Jordan inversion with pivoting on the Caltech Mark II hupercube ", Third Conf. on Hypercube Concurrent Computers and Applications, ACM, 1988, pp.1621-1634.

[13] G.A. Geist and Michael T. Heath, "Matrix factorization on a hypercube multiprocessor ", Hypercube Multiprocessors 1986, SIAM, (Ed. M.T. Heath, 1986), pp.161-180. 
[14] Cleve Moler, "Matrix computation on distributed memory multiprocessors", Hypercube Multiprocessors 1986, SIAM, (Ed. M.T. Heath, 1986), pp.181-195.

[15] C.H. Romine, "The Parallel solution of triangular system on a hypercube ", Hypercube Multiprocessors 1987, SIAM, (Ed. M.T. Heath, 1987), pp.552-559.

[16] S. Lakshmivarahan and Sudarshan .K. Dhall, "A lower bound on the communication complexity of solving linear tridiagonal systems on cube architectures ", Hypercube Muliiprocessors 1987, SIAM, (Ed. M.T. Heath, 1987), pp.560-568.

[17] Apost;olos Gerasoulis and Izzy Nelken, "Scheduling linear algebra parallel algorithms on MIMD Architectures ", Proceedings of the 4th SIAM Conference of Parallel Proctssing for Scientific Computing, 1989, pp.68-95.

[18] U. Meier, "A parallel partition method for solving banded systems of linear equations", Parallel Computing , 2, 1985, pp.33-43.

[19] S. Lemnart Johnsson, "Solving tridiagonal systems on ensemble architectures ", SIAM Journal of Scientific and Statistical Computing, Vol.8, No.3, May 1987, pp.354-392.

[20] M.D. Levin and D.J. Evans, "The inversion of matrices by the double-bordering algorithm on MIMD computers ", Parallel Computing, 17, 1991, pp.591-602.

[21] Alan Geroge, Joseph W.H. Liu and Esmond Ng, "Communication results for parallel sparse Cholesky factorization on a hypercube ", Parallel Computing, 10, 1989, pp.287-298.

[22] S. Lerinart Johnsson, "Communication efficient basic linear algebra computations on hypercube architectures", Journal of Parallel And Distributed Computing , 4, 1987, pp.133-172.

[23] Robert F. Lucas, Tom Blank and Jerome J. Tiemann, "A parallel solution method for large sparse! systems of equations ", IEEE Transactions on Computer-Aided Design, Vol.CAD-6, No.6, Nov 1987, pp.981-991.

[24] Ching-Tien Ho, "Optimal broadcasting on SIMD hypercubes without indirect addressing capability", Journal of Parallel and Distributed Computing, 13, 1991, pp.246-255.

[25] Alan George, Michael T. Heath, Joseph Liu and Esmond Ng, "Sparse Cholesky factorization on a local-memory multiprocessor ", SIAM Journal of Scientific and Statistical Compuiing, Vol.9, No.2, Mar 1988, pp.327-340.

[26] John U. Gustafson, Gary R. Montry and Robert E. Benner, "Development of parallel methods for a 1024-processor hypercube ", SIAM Journal of Scientific and Statistical Compuiing, Vol.9, No.4, Jul 1988, pp.609-638. 
[27] Anshul Gupta and Vipin Kumar, "Scalability of parallel algorithms for matrix multiplication ", Technical Report TR91-54, Computer Science Department, University of Minnesota.

[28] Anshul Gupta and Vipin Kumar, "Scalability of FFT on parallel computers ", IEEE Transactions on Parallel and Distributed Systems, Vol.4, No.8, Aug 1993, pp.922-932.

[29] Ananth Grama, Anshul Gupta and Vipin Kumar, "Isoefficiency measuring the scalability of parallel algorithms and architectures ", IEEE Parallel and Distributed Technology, Vol.1, No.3, Aug1993, pp.12-21.

[30] X-H Sun and D.T. Rover, "Scalability of parallel algorithm-Machine combinations ", IEEE Transactions on Parallel and Distributed Systems, Vol.5, No.6, Jun 1994, pp.599-613.

[31] T.von Eicken, D.E. Culler, S.C. Goldstein and K.E. Schauser, "Active messages: a mechanism for integrated communication and computation ", Proc. of the 19th Int'1 Symp. on Computer Architecture, Australia, May 1992, pp.256-266.

[32] A. Gursoy and L.V. Kale, "Dagger: combining benefits of synchronous and asynchronous communication styles ", Proc. of the $8 t h$ Int' $l$ Parallel Processing Symp., Mexico, 1994, pp.590596.

[33] W.Dally and et al., "Architecture of a message-driven processor ", Proc. of the 14th Annual Int 1 Symp. on Comp. Arch., June 1987, pp.189-196.

[34] G.M. Papadopoulos and D.E. Culler, "Monsoon: an explicit token-store architecture ", Proc. of the 17th Annual Int'l Symp. on Comp. Arch., Seattle, Washington, May 1990. 